Published By Institute of Physics Publishing for SISSA/ISAS

RECEIVED: June 17, 2004

ACCEPTED: July 16, 2004

\title{
Majorana and Majorana-Weyl fermions in lattice gauge theory
}

\author{
Teruaki Inagaki \\ Graduate School of Science and Engineering, Ibaraki University \\ Mito 310-8512, Japan \\ E-mail: teruaki@serra.sci.ibaraki.ac.jp \\ Hiroshi Suzuki \\ Institute of Applied Beam Science, Ibaraki University \\ Mito 310-8512, Japan \\ E-mail: hsuzuki@mx.ibaraki.ac.jp
}

ABSTRACT: In various dimensional euclidean lattice gauge theories, we examine a compatibility of the Majorana decomposition and the charge conjugation property of lattice Dirac operators. In $8 n$ and $1+8 n$ dimensions, we find a difficulty to decompose a classical lattice action of the Dirac fermion into a system of the Majorana fermion and thus to obtain a factrized form of the Dirac determinant. Similarly, in $2+8 n$ dimensions, there is a difficulty to decompose a classical lattice action of the Weyl fermion into a system of the Majorana-Weyl fermion and thus to obtain a factrized form of the Weyl determinant. Prescriptions based on the overlap formalism do not remove these difficulties. We argue that these difficulties are reflections of the global gauge anomaly associated to the real Weyl fermion in $8 n$ dimensions. For this reason (besides other well-known reasons), a lattice formulation of the $N=1$ super Yang-Mills theory in these dimensions is expected to be extremely difficult to find.

Keywords: Field Theories in Higher Dimensions, Field Theories in Lower Dimensions, Lattice Gauge Field Theories, Anomalies in Field and String Theories. 


\section{Contents}

1. Introduction 1

2. Majorana decomposition in even dimensional space

2.1 Majorana decomposition

2.2 Impossibility of the Majorana decomposition for $d=8 n$

3. Majorana-Weyl decomposition in $2+8 n$ dimensions 7

3.1 Definition of Weyl fermions in lattice gauge theory 8

3.2 Difficulty of the Majorana-Weyl decomposition 8

4. Majorana-Weyl fermions based on the overlap formalism 12

4.1 Brief overview of the overlap formalism

4.2 Overlap formulation of Majorana-Weyl fermions $\overline{13}$

5. Odd dimensional cases

5.1 Majorana decomposition 16

5.2 Comment on a prescription based on the overlap formalism 17

6. Physical meaning of difficulties in $8 n, 1+8 n$ and $2+8 n$ dimensions 19

A. Representation of the Clifford algebra in $d$ dimensional euclidean space 21

B. Charge conjugation properties of lattice Dirac operators 22

G. Positivity of the Majorana pfaffian in $2+8 n$ and $4+8 n$ dimensions 23

\section{Introduction}

In $d$ dimensional Minkowski spacetime, when $d=0,1,2,3,4 \bmod 8$, it is possible to define Majorana fermions by imposing the Majorana reality condition on the Dirac fermion. Also, when $d=2 \bmod 8$, it is possible to impose the Majorana condition on the Weyl fermion to define Majorana-Weyl fermions. In these dimensions, Majorana and Majorana-Weyl fermions form an irreducible spinor representation of the Lorentz group and, for this reason, these fermions are fundamental in field theories in lower and higher dimensions, especially in supersymmetric ones. In this paper, we study a possibility to formulate these degrees of freedom in euclidean lattice gauge theory. We take the Wilson-Dirac operator [1] and the overlap-Dirac operator [2] and examine a compatibility of the euclidean Majorana decomposition and the charge conjugation property of these lattice Dirac operators. ${ }^{1}$

\footnotetext{
${ }^{1}$ We take the Wilson-Dirac operator because it is the simplest Dirac operator which represents a single Dirac fermion and the overlap-Dirac operator because of its virtue concerning the chiral symmetry. A similar analysis could be performed for the Dirac operator in the perfect action approach [3].
} 
Let us first explain what we mean by Majorana and Majorana-Weyl fermions in an euclidean theory by illustrating how the euclidean Majorana decomposition works in unregularized continuum theory. If one switches the lorentzian signature to the euclidean one, the Majorana reality condition cannot be imposed in an $\mathrm{SO}(d)$ invariant manner, when $d=3$, $4 \bmod 8$, and the Majorana-Weyl condition cannot be imposed when $d=2 \bmod 8$. Instead imposing these conditions in euclidean theory, one can define Majorana and MajoranaWeyl degrees of freedom in the following way. This prescription corresponds to, in a level of the functional integral, the prescription of ref. 沟. ${ }^{2}$ Take the Dirac fermion defined by the action

$$
S=\int \mathrm{d}^{d} x \bar{\psi}(x) \gamma_{\mu} D_{\mu} \psi(x),
$$

where $D_{\mu}=\partial_{\mu}+A_{\mu}^{a} R\left(T^{a}\right)$ is the covariant derivative with respect to a gauge-group representation $R$. For Majorana fermions to be defined, the representation matrix $R\left(T^{a}\right)$ must be real, $R\left(T^{a}\right)^{*}=R\left(T^{a}\right)=-R\left(T^{a}\right)^{T}$, and this will be assumed throughout this paper when considering Majorana and Majorana-Weyl fermions. See appendix A for our convention on gamma matrices. We then introduce the Majorana decomposition by

$$
\psi=\frac{1}{\sqrt{2}}(\chi+i \eta), \quad \bar{\psi}=\frac{1}{\sqrt{2}}\left(\chi^{T} B-i \eta^{T} B\right),
$$

where $B$ denotes the charge conjugation matrix, either $B_{1}$ or $B_{2}$ in eq. (A.10) depending on the dimension $d$; for $d=0,1,2,3,4 \bmod 8$, we take, $B_{1}, B_{1}, B_{1}$ or $B_{2}, B_{1}$ and $B_{2}$, respectively. From basic properties of the charge conjugation matrices, eqs. (A.4), (A.11) and $(\mathrm{A.16})$, one finds ${ }^{3}$

$$
\left[B \gamma_{\mu} D_{\mu}(x, y)\right]^{T}=-B \gamma_{\mu} D_{\mu}(x, y) .
$$

Therefore, after substituting eq. (1.2) into eq. (1.1), one has

$$
S=\int \mathrm{d}^{d} x\left[\frac{1}{2} \chi^{T}(x) B \gamma_{\mu} D_{\mu} \chi(x)+\frac{1}{2} \eta^{T}(x) B \gamma_{\mu} D_{\mu} \eta(x)\right] .
$$

A system of the Dirac fermion is thus decomposed into two mutually independent systems, each of which may be interpreted as representing a single Majorana fermion. We refer to this type of decomposition as the Majorana decomposition. The functional integral over $\chi$, for example, then gives rise to the pfaffian $\operatorname{Pf}\left(B \gamma_{\mu} D_{\mu}\right)$ that may be regarded as the squareroot of the Dirac determinant $\operatorname{det}\left(\gamma_{\mu} D_{\mu}\right)$. It is also confirmed that for $d=5,6,7 \bmod 8$, for which there is no Majorana fermions in the Minkowski spacetime, neither $B_{1}$ nor $B_{2}$ satisfies eq. $(1.3) \cdot{ }^{4}$

When $d$ is even, one can define the (left-handed) Weyl fermion by setting the chirality constraint

$$
P_{-} \psi=\psi, \quad \bar{\psi} P_{+}=\bar{\psi}, \quad P_{ \pm}=\frac{1 \pm \gamma}{2},
$$

\footnotetext{
${ }^{2}$ For a general treatment of spinors in euclidean theory, see ref. [5].

${ }^{3}$ In this expression, $D_{\mu}(x, y)=D_{\mu} \delta^{d}(x-y)$ is the kernel of the covariant derivative in position space. The transpose operation $T$ acts also on position-space indices as $x \leftrightarrow y$.

${ }^{4}$ For $d$ dimensional Minkowski spacetime, when $d=2,3$ and $4 \bmod 8$, the (single) Majorana fermion can acquire a mass. The euclidean Majorana decomposition of the Dirac mass term $m \bar{\psi}(x) \psi(x)$ works precisely at these dimensions.
} 


\begin{tabular}{|c|c|c|c|c|c|c|c|c|c|c|c|}
\hline$d$ & 2 & 3 & 4 & 5 & 6 & 7 & 8 & 9 & 10 & 11 & 12 \\
\hline \hline Dirac & $\bigcirc$ & $\bigcirc$ & $\bigcirc$ & $\bigcirc$ & $\bigcirc$ & $\bigcirc$ & $\bigcirc$ & $\bigcirc$ & $\bigcirc$ & $\bigcirc$ & $\bigcirc$ \\
\hline Majorana & $\bigcirc$ & $\bigcirc$ & $\bigcirc$ & - & - & - & $\times$ & $\times$ & $\bigcirc$ & $\bigcirc$ & $\bigcirc$ \\
\hline Weyl & $\triangle$ & - & $\triangle$ & - & $\triangle$ & - & $\triangle$ & - & $\triangle$ & - & $\triangle$ \\
\hline Majorana-Weyl & $\times$ & - & - & - & - & - & - & - & $\times$ & - & - \\
\hline
\end{tabular}

Table 1: Euclidean lattice formulation of a single fermion in various dimensions $d$. We are not interested in those entries filled by - Entries with $\bigcirc$ can be formulated quantum mechanically as well as classically. Entries with $\triangle$ can be formulated at least classically; a consistency in the quantum level must be examined separately. For entries with $\times$, we could not find even a "classical level" formulation. The pattern repeats for $d$ modulo 8 .

in eq. (1.1). For $d=2 \bmod 8$, one may further apply the Majorana decomposition (1.2) with either use of $B_{1}$ or $B_{2}$, because $P_{-} \chi=\chi\left(\right.$ or $\left.P_{-} \eta=\eta\right)$ implies $\chi^{T} B P_{+}=\chi^{T} B$ (or $\eta^{T} B P_{+}=\eta^{T} B$ ) from the property of the charge conjugation matrices (A.6) in these dimensions. Again, this consistent chirality for $\chi($ or $\eta$ ) is possible only when $d=2 \bmod 8$. Then $\chi$, for example, may be regarded as representing the Majorana-Weyl fermion. The functional integral over $\chi$ results in the pfaffian $\operatorname{Pf}\left(B \gamma_{\mu} D_{\mu} P_{-}\right)$that may be regarded as the fourth-root of the Dirac determinant.

As we have observed, the Majorana decomposition works perfectly in unregularized gauge theories. However, it is not obvious if it works also with non-perturbative regularization, such as the lattice gauge theory. Results of our analysis in this paper can be summarized as table 1. We will find, somewhat curiously, $8 n, 1+8 n$ and $2+8 n$ dimensions refuse the Majorana and the Majorana-Weyl decompositions, even if one allows a wide class of "lattice modifications" of charge conjugation matrices and the chirality projection. ${ }^{5}$ Phenomenologically, what we will find is a conflict between the Majorana decomposition and the charge conjugation property of lattice Dirac operators. An analogous phenomenon has been known in 4 dimensional euclidean lattice theories [6, 7] as a conflict between chiral invariant Yukawa couplings and the Majorana decomposition. The fact that CP symmetry is not manifest [8, 9] in 4 dimensional lattice chiral gauge theories is also related to this conflict.

The overlap-Dirac operator was originally discovered [10, 2] from the Dirac determinant obtained by the overlap formalism [11]. In the context of the latter formalism, a definition of Majorana and Majorana-Weyl fermions has been studied in ref. [12] and in ref. [13]. See also refs. [14, 10]. According to these analyses, many-body hamiltonians, from which lowest-energy states in the overlap formalism are defined, are decomposed into two mutually independent systems; this is analogous to the Majorana decomposition. We will illustrate, however, this decomposition itself does not imply a factorized form (such as the pfaffian) of the Dirac and the Weyl determinants obtained by the overlap formalism. The overlap formalism does not remove the above difficulties.

\footnotetext{
${ }^{5}$ The $d=1$ case is special: when the gauge-group representation is real, a lattice Dirac determinant is a constant being independent of a gauge-field configuration. The partition function of the Majorana fermion can be defined as the square-root of this constant.
} 
Are these difficulties in $8 n, 1+8 n$ and $2+8 n$ dimensions merely technical ones peculiar to lattice Dirac operators that we have studied? We do not think so. We will argue that these difficulties are reflections of the global gauge anomaly [15, 16] associated to Weyl fermions belonging to a real representation in $8 n$ dimensional gauge theories. This is interesting because an elementary kinematical analysis in lattice formulation (that we will do) indicates a subtle phenomenon in quantum theory, just like the species doubling can be regarded as a reflection of the chiral anomaly. Namely, in the lattice formulation, classical theory and quantum theory are indistinguishable to some extent. On the other hand, this argument suggests that it is extremely difficult to avoid these difficulties of the Majorana and the Majorana-Weyl decompositions in these dimensions, because they have a physical origin.

This paper is organized as follows. In section 2, the Majorana decomposition in even dimensional spaces is considered. We find that $8 n$ dimensions refuse the Majorana decomposition, even if a wide class of "lattice modifications" of the charge conjugation matrix is allowed. In section 3, the Majorana decomposition of Weyl fermions is analyzed. In these sections, the overlap-Dirac operator is taken because, being a solution to the Ginsparg-Wilson relation [17], it has a nice property concerning the chiral symmetry. Weyl fermions on the lattice cannot be defined even classically without this lattice chiral symmetry [3, 18, 19]. We find that a consistent Majorana decomposition of Weyl fermions is impossible even a wide class of lattice modifications of the chirality projection and the charge conjugation matrix is allowed. In section \&, we examine somewhat in detail a prescription for Majorana-Weyl fermions in the overlap formalism of ref. [12]. In section 5, the Majorana decomposition in odd dimensional spaces is considered. We do not find a successful Majorana decomposition in $1+8 n$ dimensions. In section 6, we present an argument that difficulties of the Majorana and the Majorana-Weyl decompositions in $8 n, 1+8 n$ and $2+8 n$ dimensions have an intrinsic meaning as a reflection of the global gauge anomaly. Appendix $\mathrm{A}$ is for our conventions for gamma matrices. Charge conjugation properties of the Wilson-Dirac and the overlap-Dirac operators are summarized in appendix B. In appendix Q, we demonstrate that the Majorana pfaffian defined by the overlap-Dirac operator in $2+8 n$ and $4+8 n$ dimensions is a non-negative function of a gauge-field configuration. The lattice spacing will be denoted by $a$ throughout this paper.

\section{Majorana decomposition in even dimensional space}

\subsection{Majorana decomposition}

Here we consider a compatibility between the euclidean Majorana decomposition and the charge conjugation property of the overlap-Dirac operator (B.5) in even $d$ dimensional lattice gauge theory. The overlap-Dirac operator satisfies the gamma-hermiticity

$$
D^{\dagger}=\gamma D \gamma
$$

and the Ginsparg-Wilson relation

$$
\gamma D+D \gamma=a D \gamma D
$$

The operator is also local for "admissible" gauge-field configurations [20]. 
What we mean by the Majorana decomposition is a decomposition of the action ${ }^{6}$ of the Dirac fermion

$$
S_{\mathrm{F}}=a^{d} \sum_{x} \bar{\psi}(x) D \psi(x),
$$

into two independent systems. Imitating the continuum case (1.2), we first set

$$
\psi=\frac{1}{\sqrt{2}}(\chi+i \eta), \quad \bar{\psi}=\frac{1}{\sqrt{2}}\left(\chi^{T} B-i \eta^{T} B\right),
$$

where $B$ denotes either $B_{1}$ or $B_{2}$. For the overlap-Dirac operator $D$, from eq. (B.6), one finds ${ }^{7}$

$$
\begin{array}{ll}
\left(B_{2} D\right)^{T}=+B_{2} D, & \text { for } d=8 n, \\
\left(B_{1} D\right)^{T}=-B_{1} D, & \text { for } d=2+8 n, \\
\left(B_{2} D\right)^{T}=-B_{2} D, & \text { for } d=4+8 n .
\end{array}
$$

Therefore, for $d=2+8 n$ and for $d=4+8 n$, the action is decomposed as

$$
S_{\mathrm{F}}=a^{d} \sum_{x}\left[\frac{1}{2} \chi^{T}(x) B D \chi(x)+\frac{1}{2} \eta^{T}(x) B D \eta(x)\right]
$$

by using $B=B_{1}$ and $B=B_{2}$, respectively. However, for $d=8 n$, the above simple prescription for the Majorana decomposition does not work. We recall that the Majorana decomposition worked in unregularized continuum euclidean theory also for $d=8 n$, because $\left(B_{1} \gamma_{\mu} D_{\mu}\right)^{T}=-B_{1} \gamma_{\mu} D_{\mu}$ holds; one can use $B_{1}$ instead of $B_{2}$ in these dimensions. For the overlap-Dirac operator, on the other hand, $\left(B_{1} D\right)^{T} \neq-B_{1} D$ and this is impossible.

For $d=2+8 n$ and for $d=4+8 n$, quantum theory of the Majorana fermion can thus be defined by the functional integral

$$
\langle\mathcal{O}\rangle_{\mathrm{M}}=\int \prod_{x} \mathrm{~d} \chi(x) \mathcal{O} \exp \left[-a^{d} \sum_{x} \frac{1}{2} \chi^{T}(x) B D \chi(x)\right],
$$

in particular, the partition function is given by

$$
\langle 1\rangle_{\mathrm{M}}=\int \prod_{x} \mathrm{~d} \chi(x) \exp \left[-a^{d} \sum_{x} \frac{1}{2} \chi^{T}(x) B D \chi(x)\right]=\operatorname{Pf}(B D) .
$$

We emphasize that this construction is "perfect" in a sense that it is given by the functional integral of a local action and the fermion partition function is a smooth (single-valued) gauge invariant function of a gauge-field configuration. In appendix C, we show that the pfaffian (2.10) is moreover non-negative. Since the above provides a perfect definition of the theory, one can conclude that Majorana fermions in $2+8 n$ and $4+8 n$ dimensions are

\footnotetext{
${ }^{6}$ In this paper, we consider only the kinetic term of fermions. If other terms such as Yukawa couplings exist, the chiral symmetry for example may give rise to further restrictions [6].

${ }^{7}$ Throughout this paper, the transpose and the conjugate operations on an operator are defined with respect to the corresponding kernel in position space.
} 
free from any pathologies in quantum theory, such as the global gauge anomaly. A special case $d=4$ has been studied in ref. [21]. See also refs. [22, 23]. We note that a similar analysis as above for the Wilson-Dirac operator (B.2) leads to an identical conclusion; $d=2+8 n$ and $d=4+8 n$ cases are possible (besides a potential problem of a positivity of the pfaffian) but $d=8 n$ are not. In fact, in the context of supersymmetric theories, a number of numerical simulations for Majorana fermions in 2 and 4 dimensions have been performed by using the Wilson-Dirac operator 24].

\subsection{Impossibility of the Majorana decomposition for $d=8 n$}

In the preceding subsection, we found that the simple Majorana decomposition (2.4) does not work for $d=8 n$. Actually, under some assumptions, it can be shown that there is no possible "lattice modification" of eq. (2.4) which leads to a successful decomposition. To show this, we set

$$
\psi=\frac{1}{\sqrt{2}}(\chi+i \eta), \quad \bar{\psi}=\frac{1}{\sqrt{2}}\left(\chi^{T} B_{1} X-i \eta^{T} B_{1} X\right),
$$

where $X$ represents a possible modification of the charge conjugation matrix in lattice theory, $X=1+O(a)$. We allow $X$ to depend on the gauge field through the overlap-Dirac operator

$$
X=x(a D),
$$

where a regular function $x$ takes bounded values for all eigenvalues of the operator $a D$. For a classification of the function $x$, it is convenient to introduce operators

$$
H=\gamma D,
$$

and

$$
\Gamma=\gamma\left(1-\frac{a}{2} D\right)=\gamma-\frac{a}{2} H
$$

(These $H$ and $\Gamma$ are hermitean, because of the gamma-hermiticity (2.1).) An important property of $\Gamma$ which follows from the Ginsparg-Wilson relation (2.2) is

$$
\Gamma H+H \Gamma=0 .
$$

We also define

$$
\tilde{X}=\gamma X \gamma
$$

Then by noting relations, $\gamma=\Gamma+a H / 2, a D=\Gamma a H+a^{2} H^{2} / 2$ and $\Gamma^{2}=1-a^{2} H^{2} / 4$, the above assumption (2.12) can equivalently be expressed as

$$
\tilde{X}=h\left(a^{2} H^{2}\right)+\Gamma a H k\left(a^{2} H^{2}\right),
$$

where regular functions $h$ and $k$ take bounded values for all eigenvalues of the operator $a^{2} H^{2}$.

Now, for the Majorana decomposition (2.11) to work, we have to have $\left(B_{1} X D\right)^{T}=$ $-B_{1} X D$ or

$$
\left(B_{2} \tilde{X} H\right)^{T}=-B_{2} \tilde{X} H \text {. }
$$


Then by substituting eq. (2.17) into this expression and by using relations, $H^{T}=B_{1} H B_{1}^{-1}$ and $\Gamma^{T}=B_{1} \Gamma B_{1}^{-1}$ and eq. (2.15), it is easy to see that

$$
a^{2} H^{2}\left[h\left(a^{2} H^{2}\right)+2 \Gamma^{2} k\left(a^{2} H^{2}\right)\right]=0,
$$

and thus

$$
\tilde{X}=-2 \gamma \Gamma k\left(a^{2} H^{2}\right) .
$$

At this point, we note that the operator $\Gamma$ always has a mode such that $\Gamma \varphi=0$ (and thus $a H \varphi= \pm 2 \varphi$ ) in the free theory with an infinite lattice volume. For the overlap-Dirac operator, this may be seen from the explicit form (B.5). This property of $\Gamma$ can also be shown on more general grounds by repeating the argument of ref. [7] which invokes the Nielsen-Ninomiya theorem [25]. This shows that the kinetic term of the Majorana field $\chi$,

$$
\chi^{T} B_{1} X D \chi=\chi^{T} B_{2} \tilde{X} H \chi=-2 \chi^{T} B_{2} \gamma \Gamma k\left(a^{2} H^{2}\right) H \chi
$$

vanishes for $\chi=\varphi$. This mode $\varphi$ is thus nothing but the species doubler. We conclude that there is no possible modification $X$ which leads to a successful decomposition while being free from the species doubling.

Under some assumptions, we have observed that the Majorana decomposition does not work in $8 n$ dimensional lattice gauge theories. As a possible option, we may define the partition function of the Majorana fermion by the square-root of the Dirac determinant

$$
\langle 1\rangle_{\mathrm{M}}=\sqrt{\operatorname{det} D}
$$

However, this prescription is potentially dangerous in two aspects. First, the locality of the definition is not obvious. Namely, it is not clear if $\sqrt{\operatorname{det} D}$ can be expressed as the functional integral of a certain local (in a sense of lattice theory) action. For a recent analysis of a similar problem in the square-root prescription for the staggered fermion, see ref. 26.

Another potential problem is a smoothness (and a single-valued-ness) of the partition function as a function of a gauge-field configuration. Although $\operatorname{det} D$ is a gauge invariant real number, it is not obvious if the square-root can be defined smoothly everywhere in the configuration space of lattice gauge fields. There may occur a certain discrepancy depending on a path in the configuration space along which one defines the square-root. This phenomenon, if it occurs, is analogous to the global gauge anomaly [15, 16]. In fact, there is a good reason to believe an occurrence of this phenomenon and we will give an argument for this in a later section.

\section{Majorana-Weyl decomposition in $2+8 n$ dimensions}

As we have seen in the introduction, in unregularized continuum theory in $2+8 n$ dimensions, one can apply the Majorana decomposition on the Weyl fermion to define a system of Majorana-Weyl fermions. On the lattice, it is not trivial to define even Weyl fermions in the classical level and this has becomes possible only with recent developments [27, 11, 19], 
[28]-33]. First we briefly summarize on Weyl fermions defined by using a lattice Dirac operator which satisfies the Ginsparg-Wilson relation. Then, by using the overlap-Dirac operator, we show that a further reduction or decomposition of the Weyl fermion into Majorana-type degrees of freedom exhibits a difficulty.

\subsection{Definition of Weyl fermions in lattice gauge theory}

In lattice gauge theories, it is possible to impose the Weyl chirality condition in a consistent way, at least in the classical level, if a Dirac operator $D$ satisfies the Ginsparg-Wilson

relation (2.2) and the gamma-hermiticity (2.1). If this is the case, one can introduce a modified chiral matrix by [19]

$$
\hat{\gamma}=\gamma(1-a D), \quad \hat{\gamma}^{2}=1, \quad \hat{\gamma}^{\dagger}=\hat{\gamma},
$$

where the last two relations hold due to eqs. (2.2) and (2.1). The chirality projection operators are then defined by

$$
\hat{P}_{ \pm}=\frac{1 \pm \hat{\gamma}}{2}, \quad P_{ \pm}=\frac{1 \pm \gamma}{2} .
$$

A crucial property of $\hat{P}_{ \pm}$(which is again a result of the Ginsparg-Wilson relation) is

$$
D \hat{P}_{\mp}=P_{ \pm} D
$$

and one can consistently impose the chirality constraint

$$
\hat{P}_{-} \psi=\psi, \quad \bar{\psi} P_{+}=\bar{\psi},
$$

in the action of the Dirac fermion

$$
S_{\mathrm{F}}=a^{d} \sum_{x} \bar{\psi}(x) D \psi(x)
$$

This defines a single left-handed Weyl fermion in the classical level. We emphasize again that this consistent definition of Weyl degrees of freedom in a lattice action has become possible only after recent developments. For example, the Wilson-Dirac operator does not allow such a construction.

\subsection{Difficulty of the Majorana-Weyl decomposition}

Let us examine a validity of the Majorana decomposition for Weyl fermions by using the overlap-Dirac operator (B.5). In $2+8 n$ dimensions, one has

$$
\hat{\gamma}^{T}=-B_{2} \hat{\gamma} B_{2}^{-1}, \quad \hat{P}_{ \pm}^{T}=B_{2} \hat{P}_{\mp} B_{2}^{-1}
$$

Now, by imitating the continuum case (1.2), we may first try

$$
\psi=\frac{1}{\sqrt{2}}(\chi+i \eta), \quad \bar{\psi}=\frac{1}{\sqrt{2}}\left(\chi^{T} B-i \eta^{T} B\right),
$$


where $B$ denotes either $B_{1}$ or $B_{2}$. But this definition immediately leads to a trouble because it does not provide a consistent chirality for $\chi$ and for $\eta$; projection operators $\hat{P}_{-}$ and $P_{+}$are not exchanged under an action of $B . \hat{P}_{-\chi}=\chi$, for example, does not imply $\left(\chi^{T} B\right) P_{+}=\left(\chi^{T} B\right)$.

The above definition of the modified chiral matrix (3.1) is not unique. One may consider, for example, a one-parameter family of modified $\gamma$ matrices

$$
\gamma^{(t)}=\frac{\gamma(1-t a D)}{\sqrt{1-t(1-t) a^{2} D^{\dagger} D}}, \quad \bar{\gamma}^{(t)}=\gamma \gamma^{(1-t)} \gamma .
$$

Then relations $\left(\gamma^{(t)}\right)^{2}=\left(\bar{\gamma}^{(t)}\right)^{2}=1$ and $D \gamma^{(t)}=-\bar{\gamma}^{(t)} D$ hold. By using this kind of freedom in a definition of chirality projection operators $\hat{P}_{-}$and $P_{+}$, it could be possible to avoid the above difficulty. Also the Majorana decomposition could be modified by a "lattice way" as eq. (2.11). To examine these possibilities, we set

$$
\hat{P}_{-}=\frac{1-V}{2}, \quad \bar{P}_{+}=\frac{1+U}{2}, \quad V^{2}=U^{2}=1,
$$

where operators $V$ and $U$ are hermitean and we impose

$$
\hat{P}_{-} \psi=\psi, \quad \bar{\psi} \bar{P}_{+}=\bar{\psi},
$$

instead of eq. (3.4). Also we generalize the Majorana decomposition $\mathrm{as}^{8}$

$$
\psi=\frac{1}{\sqrt{2}}(\chi+i \eta), \quad \bar{\psi}=\frac{1}{\sqrt{2}}\left(\chi^{T} B_{1} X-i \eta^{T} B_{1} X\right),
$$

where $X=1+O(a)$. We assume that $U$ and $X$ have following structures

$$
U=\gamma u(a D), \quad X=x(a D),
$$

where regular functions $u$ and $x$ take bounded values for all eigenvalues of the operator $a D$. We also define

$$
\tilde{U}=\gamma U \gamma, \quad \tilde{U}^{2}=1, \quad \tilde{X}=\gamma X \gamma .
$$

Then by using operators $H$ and $\Gamma$ in eqs. (2.13) and (2.14), above assumptions (3.12) can equivalently be expressed as

$$
\tilde{U}=a H f\left(a^{2} H^{2}\right)+\Gamma g\left(a^{2} H^{2}\right), \quad \tilde{X}=h\left(a^{2} H^{2}\right)+\Gamma a H k\left(a^{2} H^{2}\right),
$$

where regular functions $f, g, h$ and $k$ take bounded values for all eigenvalues of the operator $a^{2} H^{2}$.

Now, for the Weyl projection (3.10) to be consistent in the action, we have to have $D \hat{P}_{-}=\bar{P}_{+} D$. This implies

$$
-H V=\tilde{U} H .
$$

\footnotetext{
${ }^{8}$ In this expression, we may start with $B_{2}$ instead of $B_{1}$. The following analysis can be repeated for this case also and a similar conclusion is resulted.
} 
By substituting eq. (3.14) into this, we find

$$
V=-a H f\left(a^{2} H^{2}\right)+\Gamma g\left(a^{2} H^{2}\right),
$$

by recalling eq. (2.15). Also, to ensure a consistent chirality for the Majorana field, $\hat{P}_{-\chi}=$ $\chi \Rightarrow\left(\chi^{T} B_{1} X\right) \bar{P}_{+}=\left(\chi^{T} B_{1} X\right)$, we must have (note that $\left.B_{1}=\gamma B_{2}=-B_{2} \gamma\right)$

$$
-B_{2}^{-1} V^{T} B_{2} \tilde{X}=\tilde{X} \tilde{U}
$$

This implies

$$
a H\left[h\left(a^{2} H^{2}\right) f\left(a^{2} H^{2}\right)-\Gamma^{2} k\left(a^{2} H^{2}\right) g\left(a^{2} H^{2}\right)\right]=0,
$$

by noting $H^{T}=-B_{2} H B_{2}^{-1}$ and $\Gamma^{T}=-B_{2} \Gamma B_{2}^{-1}$ in $2+8 n$ dimensions. Note that this relation shows

$$
\tilde{X} \tilde{U}=\left[h\left(a^{2} H^{2}\right) g\left(a^{2} H^{2}\right)+a^{2} H^{2} k\left(a^{2} H^{2}\right) f\left(a^{2} H^{2}\right)\right] \Gamma .
$$

Finally, for the Majorana decomposition to work, we must have $\left(B_{1} X D\right)^{T}=-B_{1} X D$ and

$$
H B_{2}^{-1} \tilde{X}^{T} B_{2}=\tilde{X} H
$$

but this requirement sets no restriction on the functions $h$ and $k$.

As noted in section 2.2, the operator $\Gamma$ has a mode such that $\Gamma \varphi=0$ and $a H \varphi= \pm 2 \varphi$. Above relations then imply

$$
\tilde{U} \varphi= \pm 2 f(4) \varphi, \quad \tilde{X} \varphi=h(4) \varphi, \quad f(4) h(4)=0,
$$

where the last relation follows from eq. (3.19). From these expressions, a trouble becomes clear: If $f(4)=0$, then we cannot set $\tilde{U}^{2}=V^{2}=1$ on $\varphi$. Namely, the projection operator cannot be properly defined. On the other hand, if $h(4)=0$, the kinetic term of the Majorana field, $\chi^{T} B_{1} X D \chi=-\chi^{T} B_{2} \tilde{X} H \chi$, vanishes for $\chi=\varphi$. This mode $\varphi$ is nothing but the species doubler. An interesting example in the latter case is the choice, $U=\gamma$, $V=\hat{\gamma}$ and $X=\gamma \Gamma$. It can be seen that this choice fulfills all the above requirements for a consistent Majorana decomposition, but the kinetic term acquires doubler's zeros.

Summarizing our analysis so far, we have observed that there is always a conflict between the Weyl projection of the type (3.10) based on the Ginsparg-Wilson relation and the modified Majorana decomposition (3.11) in $2+8 n$ dimensions.

Going back to the standard choice (3.1), to define the partition function of the Weyl fermion

$$
\langle 1\rangle_{\mathrm{W}}=\int \mathrm{D}[\psi] \mathrm{D}[\bar{\psi}] e^{-S_{\mathrm{F}}}, \quad S_{\mathrm{F}}=a^{d} \sum_{x} \bar{\psi}(x) D \psi(x)
$$

one introduces an orthonormal complete set of vectors in the constrained space (3.4),

$$
\begin{array}{ll}
\hat{P}_{-} v_{j}(x)=v_{j}(x), & \left(v_{k}, v_{j}\right)=a^{d} \sum_{x} v_{k}(x)^{\dagger} v_{j}(x)=\delta_{k j}, \\
\bar{v}_{k}(x) P_{+}=\bar{v}_{k}(x), \quad\left(\bar{v}_{k}^{\dagger}, \bar{v}_{j}^{\dagger}\right)=\delta_{k j} .
\end{array}
$$


We note

$$
\operatorname{Tr} \hat{\gamma}=\operatorname{Tr} \hat{\gamma}^{T}=-\operatorname{Tr} B_{2} \hat{\gamma} B_{2}^{-1}=-\operatorname{Tr} \hat{\gamma}=0,
$$

due to the reality of the representation, and $\operatorname{Tr} \hat{P}_{-}=\operatorname{Tr} P_{+}=(1 / 2) \operatorname{Tr} 1$. Thus indices $j$, $k$ in eqs. (3.23) and (3.24) run over from 1 to (1/2) $\operatorname{Tr} 1$ that is a fixed number being independent of a gauge-field configuration. ${ }^{9}$ By expanding fermion fields in terms of these bases

$$
\psi(x)=\sum_{j} v_{j}(x) c_{j}, \quad \bar{\psi}(x)=\sum_{k} \bar{c}_{k} \bar{v}_{k}(x)
$$

the integration measure is defined by

$$
\mathrm{D}[\psi] \mathrm{D}[\bar{\psi}]=\prod_{j} \mathrm{~d} c_{j} \prod_{k} \mathrm{~d} \bar{c}_{k} .
$$

Then the partition function $(3.22)$ is given by

$$
\langle 1\rangle_{\mathrm{W}}=\operatorname{det} M, \quad M_{k j}=a^{d} \sum_{x} \bar{v}_{k}(x) D v_{j}(x)=\frac{2}{a} a^{d} \sum_{x} \bar{v}_{k}(x) v_{j}(x) .
$$

In deriving the last expression, we have noted the relation $P_{+} D \hat{P}_{-}=(2 / a) P_{+} \hat{P}_{-}$.

We have observed that the Majorana decomposition of Weyl fermions has a difficulty. As a possible option, we may define the partition function of Majorana-Weyl fermions by the square-root of the Weyl determinant

$$
\langle 1\rangle_{\mathrm{MW}}=\sqrt{\operatorname{det} M} .
$$

However, as the square-root prescription for Majorana fermions (2.22), this prescription is potentially dangerous; the locality and the smoothness are not obvious. An analysis of smoothness in the present case is not easy, because $\operatorname{det} M$ depends on a choice of the basis vectors $\left\{v_{j}\right\}(3.23)$. A system of a single Majorana-Weyl fermion is always anomalous and one has to introduce left- and right-handed Majorana-Weyl fermions to eliminate the local (perturbative) gauge anomaly in continuum theory. Then one has to examine if it is possible to choose basis vectors such that $\operatorname{det} M$ is gauge invariant. The choice must also preserve the locality. See refs. [28, 29] for a proper way to formulate the problem. In a non-perturbative level, such an "ideal" basis has been constructed only for U(1) gauge theories 28]. ${ }^{10}$ It might be possible to study the present problem of smoothness by using real representations of $\mathrm{SO}(2)$ gauge theories.

If the matrix $M_{k j}$ in eq. (3.28) is anti-symmetric then the square-root in eq. (3.29) will be

$$
\langle 1\rangle_{\mathrm{MW}}=\operatorname{Pf} M
$$

and the above problem of smoothness is removed. Note again that, however, the matrix $M$ depends on a choice of basis vectors $\left\{v_{j}\right\}$. For a generic choice of basis vectors, the ma-

\footnotetext{
${ }^{9}$ Since $\operatorname{Tr} 1=\sum_{x} \operatorname{tr} 1$ and $\operatorname{tr} 1=2^{d / 2}$, Tr 1 is always an even positive integer.

${ }^{10}$ As a recent attempt towards an implementation of this construction in actual numerical simulations, see ref. 34. As a completely different kind of approach to lattice chiral gauge theories, see ref. [35].
} 
trix $M$ is neither anti-symmetric nor real. Again one first has to seek an ideal basis and study its property before examining if this factorization of the chiral determinant $\operatorname{det} M$ occurs.

It is interesting to note that, in the overlap formalism for real Weyl fermions in $2+8 n$ dimensions, it has been known that many-body hamiltonians, from which lowest-energy states in the overlap formalism are defined, are decomposed into two independent systems [12]. If this fact implies a simple factorization (such as the pfaffian) of the Weyl determinant, it would provide a way out from above difficulties. We will examine this possibility in the next section; our conclusion will, however, be negative.

\section{Majorana-Weyl fermions based on the overlap formalism}

\subsection{Brief overview of the overlap formalism}

First we briefly recall basics of the overlap formalism. We assume that $d=2+8 n$ and the gauge-group representation is real. In the overlap formalism, the Weyl determinant is defined as an overlap of two states $\langle L-\mid L+\rangle$, each of the states is the ground state of many-body hamiltonians $\mathcal{H}^{+}$and $\mathcal{H}^{-}$, respectively. These $\mathcal{H}^{ \pm}$are defined by

$$
\mathcal{H}^{ \pm}=a^{d} \sum_{x} a(x)^{\dagger} H^{ \pm} a(x)
$$

where hermitean Wilson-Dirac operators $H^{ \pm}$are given by

$$
H^{+}=\gamma\left(\frac{1}{a}-D_{\mathrm{W}}\right), \quad H^{-}=\gamma\left(-m-D_{\mathrm{W}}\right)
$$

The mass parameter $m$ is taken to be infinity $m \rightarrow+\infty$ in the final expressions of the overlap. The creation and annihilation operators obey the anti-commutation relations

$$
\left\{a(x), a(y)^{\dagger}\right\}=a^{-d} \delta_{x, y}, \quad\{a(x), a(y)\}=0 .
$$

To find the lowest-energy states $|L \pm\rangle$, we define eigenfunctions of $H^{ \pm}$with negative eigenvalues:

$$
H^{ \pm} v_{j}^{ \pm}(x)=-\lambda_{j}^{ \pm} v_{j}^{ \pm}(x), \quad \lambda_{j}^{ \pm}>0, \quad\left(v_{k}^{ \pm}, v_{j}^{ \pm}\right)=a^{d} \sum_{x} v_{k}^{ \pm}(x)^{\dagger} v_{j}^{ \pm}(x)=\delta_{k j} .
$$

Due to the charge conjugation property of the hermitean Wilson-Dirac operators in $2+$ $8 n$ dimensions (see eq. (B.4)), we have

$$
H^{ \pm} B_{2}^{-1} v_{j}^{ \pm}(x)^{*}=+\lambda_{j}^{ \pm} B_{2}^{-1} v_{j}^{ \pm}(x)^{*} .
$$

Therefore, eigenvalues of $H^{+}$, for example, always appear in pair as $+\lambda_{j}^{+}$and $-\lambda_{j}^{+}$. We assume that $H^{ \pm}$have no zero modes; otherwise lowest-energy states are not uniquely defined. Since $H^{ \pm}$are hermitean, a set of vectors $\left\{v_{j}^{+}, B_{2}^{-1} v_{j}^{+*}\right\}$ or $\left\{v_{j}^{-}, B_{2}^{-1} v_{j}^{-*}\right\}$ spans a complete set. These facts show that, in eq. (4.4), the index $j$ runs from 1 to (1/2) $\operatorname{Tr} 1$. 
Expanding the annihilation operators by using each of complete sets,

$$
a(x)=\sum_{j=1}^{\operatorname{Tr}(1 / 2)}\left[a_{j}^{ \pm} v_{j}^{ \pm}(x)+\tilde{a}_{j}^{ \pm} B_{2}^{-1} v_{j}^{ \pm}(x)^{*}\right],
$$

we have

$$
\left\{a_{j}^{ \pm}, a_{k}^{ \pm \dagger}\right\}=\left\{\tilde{a}_{j}^{ \pm}, \tilde{a}_{k}^{ \pm \dagger}\right\}=\delta_{j k}, \quad\left\{a_{j}^{ \pm}, a_{k}^{ \pm}\right\}=\left\{\tilde{a}_{j}^{ \pm}, \tilde{a}_{k}^{ \pm}\right\}=\left\{a_{j}^{ \pm}, \tilde{a}_{k}^{ \pm}\right\}=\left\{a_{j}^{ \pm}, \tilde{a}_{k}^{ \pm \dagger}\right\}=0,
$$

and

$$
\mathcal{H}^{ \pm}=\sum_{j=1}^{\operatorname{Tr}(1 / 2)}\left(-\lambda_{j}^{ \pm} a_{j}^{ \pm \dagger} a_{j}^{ \pm}+\lambda_{j}^{ \pm} \tilde{a}_{j}^{ \pm \dagger} \tilde{a}_{j}^{ \pm}\right) .
$$

In deriving above expressions, we have noted the fact that $v_{j}^{+}$and $B_{2}^{-1} v_{k}^{+*}$ for example are orthogonal, because they give rise to different eigenvalues of the hermitean operator $\mathrm{H}^{+}$. From this expression of hamiltonians, the lowest-energy states are given by

$$
|L+\rangle=a_{1}^{+\dagger} a_{2}^{+\dagger} \cdots a_{\operatorname{Tr}(1 / 2)}^{+\dagger}|0\rangle, \quad|L-\rangle=a_{1}^{-\dagger} a_{2}^{-\dagger} \cdots a_{\operatorname{Tr}(1 / 2)}^{-\dagger}|0\rangle .
$$

Here it is important to note the fact that the Fock vacuum $|0\rangle$ is common to $|L+\rangle$ and to $|L-\rangle$ because it is specified by the condition $a(x)|0\rangle=0$. The overlap of these two states is thus given by

$$
\begin{aligned}
\langle L-\mid L+\rangle & =\left\langle 0\left|a_{\operatorname{Tr}(1 / 2)}^{-} \cdots a_{2}^{-} a_{1}^{-} a_{1}^{+\dagger} a_{2}^{+\dagger} \cdots a_{\operatorname{Tr}(1 / 2)}^{+\dagger}\right| 0\right\rangle \\
& =\operatorname{det} m,
\end{aligned}
$$

where the matrix $m$ is given by

$$
m_{k j}=a^{d} \sum_{x} v_{k}^{-}(x)^{\dagger} v_{j}^{+}(x) .
$$

Comparing the overlap-Dirac operator $(\overline{B .5})$ and the hermitean Wilson-Dirac operators (4.2), we see that $\hat{\gamma}=H^{+} / \sqrt{\left(H^{+}\right)^{2}}$ and $\gamma=-\lim _{m \rightarrow+\infty} H^{-} / \sqrt{\left(H^{-}\right)^{2}}$. Therefore the chirality constraints (3.23) and (3.24) and the conditions for eigenfunctions (4.4) turn out to be identical; we can thus identify $v_{j}=v_{j}^{+}$and $\bar{v}_{k}^{\dagger}=v_{k}^{-}$. With this identification, $M_{k j}=(2 / a) m_{k j}$ and the chiral determinants given by eq. (3.28) and by eq. (4.10) are identical, up to a proportionality constant.

\subsection{Overlap formulation of Majorana-Weyl fermions}

Now, for $2+8 n$ dimensions, the many-body hamiltonians $\mathcal{H}^{ \pm}$enjoy a decomposition into two mutually independent systems [12]; this is analogous to the Majorana decomposition. To see this, we define hermitean parts of the creation and annihilation operators by

$$
\gamma(x)=\frac{1}{\sqrt{2}}\left[B_{2}^{1 / 2} a(x)+B_{2}^{-1 / 2} a(x)^{\dagger}\right], \quad \delta(x)=\frac{1}{\sqrt{2} i}\left[B_{2}^{1 / 2} a(x)-B_{2}^{-1 / 2} a(x)^{\dagger}\right] .
$$


Here we have introduced matrices $B_{2}^{1 / 2}$ and $B_{2}^{-1 / 2}$ as objects such that $\left(B_{2}^{1 / 2}\right)^{2}=B_{2}$, $\left(B_{2}^{-1 / 2}\right)^{2}=B_{2}^{-1}$ and $B_{2}^{1 / 2} B_{2}^{-1 / 2}=1$. Such matrices are not unique but we can adopt

$$
B_{2}^{1 / 2}=\frac{1}{\sqrt{2}}\left(1+B_{2}\right), \quad B_{2}^{-1 / 2}=\frac{1}{\sqrt{2}}\left(1-B_{2}\right),
$$

because $B_{2}^{2}=-1$ holds in our representation (see eq. (A.14)). Note that these $B_{2}^{1 / 2}$ and $B_{2}^{-1 / 2}$ are symmetric matrices. It is then easy to see that each of $\gamma(x)$ and $\delta(x)$ generates the Clifford algebra including space indices:

$$
\{\gamma(x), \gamma(y)\}=\{\delta(x), \delta(y)\}=a^{-d} \delta_{x, y}, \quad\{\gamma(x), \delta(y)\}=0 .
$$

Also, by defining

$$
H_{\mathrm{MW}}^{ \pm}=B_{2}^{1 / 2} H^{ \pm} B_{2}^{-1 / 2}
$$

and by noting $\left(H_{\mathrm{MW}}^{ \pm}\right)^{T}=-H_{\mathrm{MW}}^{ \pm}$, we have the decomposition

$$
\mathcal{H}^{ \pm}=\frac{1}{2} a^{d} \sum_{x} \gamma(x) H_{\mathrm{MW}}^{ \pm} \gamma(x)+\frac{1}{2} a^{d} \sum_{x} \delta(x) H_{\mathrm{MW}}^{ \pm} \delta(x) .
$$

Thus the many-body hamiltonians are decomposed into two mutually independent systems. One would then expect that this decomposition, after applying the overlap formula only to (say) a system of $\gamma(x)$, leads to a natural factorization of the overlap formula (4.10) (such as the pfaffian) for Weyl fermions. However, the real situation is more subtle as we will see below.

To see what happens clearly, we further expand $\gamma(x)$ by using eigenfunctions (4.4),

$$
\begin{aligned}
\gamma(x) & =B_{2}^{1 / 2} \sum_{j=1}^{\operatorname{Tr}(1 / 2)}\left[\xi_{j}^{+} v_{j}^{+}(x)+\xi_{j}^{+\dagger} B_{2}^{-1} v_{j}^{+}(x)^{*}\right] \\
& =B_{2}^{1 / 2} \sum_{j=1}^{\operatorname{Tr}(1 / 2)}\left[\xi_{j}^{-} v_{j}^{-}(x)+\xi_{j}^{-\dagger} B_{2}^{-1} v_{j}^{-}(x)^{*}\right] .
\end{aligned}
$$

Then we have

$$
\left\{\xi_{j}^{+}, \xi_{k}^{+\dagger}\right\}=\delta_{j, k}, \quad\left\{\xi_{j}^{+}, \xi_{k}^{+}\right\}=0, \quad\left\{\xi_{j}^{-}, \xi_{k}^{-\dagger}\right\}=\delta_{j, k}, \quad\left\{\xi_{j}^{-}, \xi_{k}^{-}\right\}=0,
$$

and

$$
\begin{aligned}
& \mathcal{H}_{\mathrm{MW}}^{+}=\frac{1}{2} a^{d} \sum_{x} \gamma(x) H_{\mathrm{MW}}^{+} \gamma(x)=\sum_{j=1}^{\operatorname{Tr}(1 / 2)}\left(-\lambda_{j}^{+}\right)\left(\xi_{j}^{+\dagger} \xi_{j}^{+}-\frac{1}{2}\right), \\
& \mathcal{H}_{\mathrm{MW}}^{-}=\frac{1}{2} a^{d} \sum_{x} \gamma(x) H_{\mathrm{MW}}^{-} \gamma(x)=\sum_{j=1}^{\operatorname{Tr}(1 / 2)}\left(-\lambda_{j}^{-}\right)\left(\xi_{j}^{-\dagger} \xi_{j}^{-}-\frac{1}{2}\right) .
\end{aligned}
$$

From these expressions, the lowest-energy states of these "Majorana-Weyl" hamiltonians are given by

$$
\begin{aligned}
|L+\rangle_{\mathrm{MW}} & =\xi_{1}^{+\dagger} \xi_{2}^{+\dagger} \cdots \xi_{\operatorname{Tr}(1 / 2)}^{+\dagger}|0\rangle_{+}, \\
|L-\rangle_{\mathrm{MW}} & =\xi_{1}^{-\dagger} \xi_{2}^{-\dagger} \cdots \xi_{\operatorname{Tr}(1 / 2)}^{-\dagger}|0\rangle_{-}
\end{aligned}
$$


The important point to recognize here is that the Fock vacua, $|0\rangle_{+}$and $|0\rangle_{-}$, are generally different; the former is defined by the condition $\xi_{j}^{+}|0\rangle_{+}=0$ and the latter is defined by $\xi_{j}^{-}|0\rangle_{-}=0$. Since $\xi_{j}^{+}$and $\xi_{j}^{-}$are related by a non-trivial Bogoliubov transformation as we will see shortly, a relation between $|0\rangle_{+}$and $|0\rangle_{-}$is also non-trivial. This is a crucial difference from the overlap formula for Weyl fermions. Due to this fact, there emerge non-polynomial dependences of the Majorana-Weyl overlap ${ }_{\mathrm{MW}}\langle L-\mid L+\rangle_{\mathrm{MW}}$ on eigenfunctions (4.4).

The Bogoliubov transformation is given by

$$
\xi_{k}^{-}=\sum_{j=1}^{\operatorname{Tr}(1 / 2)}\left(m_{k j} \xi_{j}^{+}+n_{k j} \xi_{j}^{+\dagger}\right)
$$

where

$$
m_{k j}=a^{d} \sum_{x} v_{k}^{-}(x)^{\dagger} v_{j}^{+}(x), \quad n_{k j}=a^{d} \sum_{x} v_{k}^{-}(x)^{\dagger} B_{2}^{-1} v_{j}^{+}(x)^{*} .
$$

These matrices satisfy

$$
\begin{aligned}
m m^{\dagger}+n n^{\dagger} & =1, \\
m n^{T}+n m^{T} & =0,
\end{aligned}
$$

to ensure the identical anti-commutation relations (4.18) for $\xi_{j}^{+}$and for $\xi_{j}^{-}$. The Bogoliubov transformation can equally be represented as

$$
\xi_{k}^{-}=e^{-\mathcal{G}} \sum_{j=1}^{\operatorname{Tr}(1 / 2)} m_{k j} \xi_{j}^{+} e^{\mathcal{G}}, \quad \mathcal{G}=\frac{1}{2} \sum_{j, k} \xi_{j}^{+\dagger}\left(m^{-1} n\right)_{j k} \xi_{k}^{+\dagger}
$$

where this construction of $\mathcal{G}$ is consistent because the matrix $m^{-1} n$ is anti-symmetric from eq. (4.26). Two Fock vacua are thus related as

$$
|0\rangle_{-}=C e^{-\mathcal{G}}|0\rangle_{+}
$$

and the normalization constant $C$ is determined by ${ }^{11}$

$$
\begin{aligned}
1 & =|C|^{2}+\left\langle 0\left|e^{-\mathcal{G}^{\dagger}} e^{-\mathcal{G}}\right| 0\right\rangle_{+} \\
& =|C|^{2}{ }_{+}\left\langle 0\left|\exp \left[\frac{1}{2} \sum_{j, k} \xi_{j}^{+}\left(m^{-1 *} n^{*}\right)_{j k} \xi_{k}^{+}\right] \exp \left[-\frac{1}{2} \sum_{l, m} \xi_{l}^{+\dagger}\left(m^{-1} n\right)_{l m} \xi_{m}^{+\dagger}\right]\right| 0\right\rangle_{+} \\
& =|C|^{2} \operatorname{det}^{1 / 2}\left(1-m^{-1 *} n^{*} m^{-1} n\right) .
\end{aligned}
$$

\footnotetext{
${ }^{11}$ In deriving the last expression, we have used the formula which holds for fermionic creation and annihilation operators,

$$
\left\langle 0\left|\exp \left(\frac{1}{2} a \mathcal{M} a\right) \exp \left(\frac{1}{2} a^{\dagger} \mathcal{N} a^{\dagger}\right)\right| 0\right\rangle=\operatorname{det}^{1 / 2}(1+\mathcal{M N}) .
$$

This formula may readily be proven by going to the coherent state representation.
} 
We next note

$$
\begin{aligned}
\operatorname{det}\left(1-m^{-1 *} n^{*} m^{-1} n\right) & =\operatorname{det}\left(1+m^{-1 *} n^{*} n^{T} m^{-1 T}\right) \\
& =\operatorname{det}\left(1+m^{-1} n n^{\dagger} m^{-1 \dagger}\right) \\
& =\operatorname{det}\left[m^{-1}\left(m m^{\dagger}+n n^{\dagger}\right) m^{-1 \dagger}\right] \\
& =|\operatorname{det} m|^{-2},
\end{aligned}
$$

where the last equality follows from eq. (4.25). Therefore we have

$$
C=|\operatorname{det} m|^{1 / 2} e^{i \theta}
$$

where $\theta$ is an unspecified phase which may depend on a gauge-field configuration. The Majorana-Weyl overlap thus gives rise to

$$
\begin{aligned}
\mathrm{MW}\langle L-\mid L+\rangle_{\mathrm{MW}} & ={ }_{-}\left\langle 0\left|\xi_{\operatorname{Tr}(1 / 2)}^{-} \cdots \xi_{2}^{-} \xi_{1}^{-} \xi_{1}^{+\dagger} \xi_{2}^{+\dagger} \cdots \xi_{\operatorname{Tr}(1 / 2)}^{+\dagger}\right| 0\right\rangle_{+} \\
& =C^{*}{ }_{+}\left\langle 0\left|e^{-\mathcal{G}^{\dagger}} e^{-\mathcal{G}} m_{\operatorname{Tr}(1 / 2) j} \xi_{j}^{+} \cdots m_{1 k} \xi_{k}^{+} e^{\mathcal{G}} \xi_{1}^{+\dagger} \xi_{2}^{+\dagger} \cdots \xi_{\operatorname{Tr}(1 / 2)}^{+\dagger}\right| 0\right\rangle_{+} \\
& =C^{*} \operatorname{det} m_{+}\left\langle 0\left|e^{-\mathcal{G}^{\dagger}} e^{-\mathcal{G}}\right| 0\right\rangle_{+} \\
& =C^{-1} \operatorname{det} m \\
& =|\operatorname{det} m|^{1 / 2} e^{i \vartheta}, \quad \vartheta=\arg \operatorname{det} m-\theta .
\end{aligned}
$$

We have described the calculation somewhat in detail to illustrate how non-polynomial dependences on the eigenfunctions emerge. The final result can be regarded as

$$
{ }_{\mathrm{MW}}\langle L-\mid L+\rangle_{\mathrm{MW}}=\sqrt{\operatorname{det} m},
$$

where a definition of the square-root amounts to specifying how the phase $\vartheta$ changes according to a change of gauge fields. This result is certainly ensuring because a square of the Majorana-Weyl overlap is the Weyl overlap. Nevertheless, the result is not interesting because the Majorana-Weyl overlap eventually reduces to the square-root prescription (3.29). The difficulty we faced in the previous section is not removed by the overlap formalism.

\section{Odd dimensional cases}

\subsection{Majorana decomposition}

In this section, we consider the Majorana decomposition in odd dimensional spaces. For $1+8 n$ dimensions, as shown in eqs. $(\overline{B .3})$ and $(\overline{B .6})$, both the Wilson-Dirac operator and the overlap-Dirac operator have no simple transformation law under the transpose operation. Hence the Majorana decomposition cannot be applied. Even we cannot study possible "lattice modifications" of the charge conjugation matrix, due to lack of a definite charge conjugation property of these lattice Dirac operators.

For $3+8 n$ dimensions, the Wilson-Dirac operator has the desired property

$$
\left(B_{1} D_{\mathrm{W}}\right)^{T}=-B_{1} D_{\mathrm{W}}
$$


Therefore, the Majorana decomposition can be applied at least for the Wilson-Dirac operator. Namely

$$
\langle 1\rangle_{\mathrm{M}}=\int \prod_{x} \mathrm{~d} \chi(x) \exp \left[-a^{d} \sum_{x} \frac{1}{2} \chi^{T}(x) B_{1} D_{\mathrm{W}} \chi(x)\right]=\operatorname{Pf}\left(B_{1} D_{\mathrm{W}}\right) .
$$

This immediately implies that a quantum theory of Majorana fermions in $3+8 n$ dimensions is free from any pathologies. Although this is a "perfect" definition, the pfaffian is neither positive nor real in general. One way to see this is to recall that the Dirac determinant det $D_{\mathrm{W}}$, being gauge invariant, suffers from the parity anomaly [36, 37]. ${ }^{12}$ From the relation

$$
D_{\mathrm{W}}(x, y)^{\dagger}=D_{\mathrm{W}}(-x,-y)\left(U \rightarrow U^{P}\right),
$$

where $U^{P}$ is the parity transformation of gauge fields,

$$
U^{P}(x, \mu)=U(-x-a \hat{\mu}, \mu)^{-1},
$$

we have

$$
\left(\operatorname{det} D_{\mathrm{W}}\right)^{*}=\operatorname{det} D_{\mathrm{W}}\left(U \rightarrow U^{P}\right) .
$$

The parity anomaly on the other hand implies

$$
\operatorname{det} D_{\mathrm{W}}\left(U^{P}\right) \neq \operatorname{det} D_{\mathrm{W}}(U) \text {. }
$$

Therefore, det $D_{\mathrm{W}}$ is a complex number. Since the square of the pfaffian $\operatorname{Pf}\left(B_{1} D_{\mathrm{W}}\right)$ is the Dirac determinant, the pfaffian cannot be a real number in general.

\subsection{Comment on a prescription based on the overlap formalism}

In ref. [13], following the overlap formalism for Dirac fermions in odd dimensions [38], it was pointed out that many-body hamiltonians in the overlap formalism enjoy a decomposition when $d=3+8 n$ (and when the gauge-group representation is real). Here, we note that this decomposition does not imply a factorized form of the Dirac determinant. The overlap formalism for the Dirac fermion in $3+8 n$ dimensions [38, 10] starts with hermitean WilsonDirac operators in a space with one dimension higher, i.e., $H^{ \pm}$in $4+8 n$ dimensions

$$
H^{ \pm}=\gamma\left( \pm m-D_{\mathrm{W}}\right)
$$

but with a "dimensional reduction" to $3+8 n$ dimensions. Here the dimensional reduction means that the summation over $\mu$ in the Wilson-Dirac operator runs from $\mu=0$ to $\mu=$ $2+8 n$ only:

$$
D_{\mathrm{W}}=\frac{1}{2} \sum_{\mu=0}^{2+8 n}\left[\gamma_{\mu}\left(\nabla_{\mu}^{*}+\nabla_{\mu}\right)-a \nabla_{\mu}^{*} \nabla_{\mu}\right]
$$

\footnotetext{
${ }^{12}$ We regard the parity anomaly as a property of the theory rather than a pathology. H.S. would like to thank Martin Lüscher for an enlightening discussion on this point.
} 
This operator does not contain gauge fields along the $\mu=3+8 n$ direction although gamma matrices are those for $4+8 n$ dimensions. The many-body hamiltonians are then defined by

$$
\mathcal{H}^{ \pm}=a^{d} \sum_{x} a(x)^{\dagger} H^{ \pm} a(x)
$$

where the position $x$ runs over a $3+8 n$ (odd) dimensional lattice. After applying the overlap prescription, this setup defines the Dirac fermion in $3+8 n$ dimensions.

To see that above many-body hamiltonians are decomposed into two independent systems, we define the matrix $B_{3}$ (note that gamma matrices are those of $4+8 n$ dimensions) by

$$
B_{3}=B_{1} \gamma_{3+8 n}, \quad B_{3}^{T}=+B_{3}, \quad B_{3}^{2}=-1 .
$$

Due to the fact that $\mu=3+8 n$ is omitted in the Wilson-Dirac operator (5.8), one has

$$
\left(H^{ \pm}\right)^{T}=-B_{3} H^{ \pm} B_{3}^{-1}
$$

Therefore, by defining

$$
B_{3}^{1 / 2}=\frac{1}{\sqrt{2}}\left(1+B_{3}\right), \quad B_{3}^{-1 / 2}=\frac{1}{\sqrt{2}}\left(1-B_{3}\right),
$$

and

$$
H_{\mathrm{M}}^{ \pm}=B_{3}^{1 / 2} H^{ \pm} B_{3}^{-1 / 2}
$$

one has $\left(H_{\mathrm{M}}^{ \pm}\right)^{T}=-H_{\mathrm{M}}^{ \pm}$. Finally, by setting

$$
\gamma(x)=\frac{1}{\sqrt{2}}\left[B_{3}^{1 / 2} a(x)+B_{3}^{-1 / 2} a(x)^{\dagger}\right], \quad \delta(x)=\frac{1}{\sqrt{2} i}\left[B_{3}^{1 / 2} a(x)-B_{3}^{-1 / 2} a(x)^{\dagger}\right],
$$

one has a decomposition of many-body hamiltonians

$$
\mathcal{H}^{ \pm}=\frac{1}{2} a^{d} \sum_{x} \gamma(x) H_{\mathrm{M}}^{ \pm} \gamma(x)+\frac{1}{2} a^{d} \sum_{x} \delta(x) H_{\mathrm{M}}^{ \pm} \delta(x) .
$$

One clearly sees a similarity of expressions to those of section 4.2 with the replacement $B_{2} \rightarrow$ $B_{3}$. In particular, since the operators $\gamma(x)$ and $\delta(x)$ obey anti-commutation relations identical to eq. (4.14), we have the same conclusion as section 4.2. An application of the overlap prescription to a system of $\gamma(x)$ reduces to the square-root prescription; this time, it is the square-root of the Dirac determinant defined by the overlap formalism.

For $1+8 n$ dimensions, we found that the Majorana decomposition does not work with either use of the Wilson-Dirac and the overlap-Dirac operators. For these dimensions, we may try a use of the overlap formalism. For $d=1+8 n$, one starts with the hermitean Wilson operator $H^{ \pm}=\gamma\left( \pm m-D_{\mathrm{W}}\right)$ in $2+8 n$ dimensions, but with the dimensional reduction in the $\mu=1+8 n$ direction:

$$
D_{\mathrm{W}}=\frac{1}{2} \sum_{\mu=0}^{8 n}\left[\gamma_{\mu}\left(\nabla_{\mu}^{*}+\nabla_{\mu}\right)-a \nabla_{\mu}^{*} \nabla_{\mu}\right] .
$$


Since the gamma matrices are those of $2+8 n$ dimensions, all algebraic relations in section 4.2 hold as they stand. This is expected because, after the dimensional reduction, Majorana-Weyl fermions in $2+8 n$ dimensions become Majorana fermions in $1+8 n$ dimensions. In particular, defining

$$
H_{\mathrm{M}}^{ \pm}=B_{2}^{1 / 2} H^{ \pm} B_{2}^{-1 / 2}
$$

and using eq. (4.12), we have a decomposition

$$
\mathcal{H}^{ \pm}=\frac{1}{2} a^{d} \sum_{x} \gamma(x) H_{\mathrm{M}}^{ \pm} \gamma(x)+\frac{1}{2} a^{d} \sum_{x} \delta(x) H_{\mathrm{M}}^{ \pm} \delta(x) .
$$

Again an application of the overlap formalism to a system of $\gamma(x)$ will reduce to the squareroot prescription and this setup does not remove a difficulty of the Majorana decomposition in $1+8 n$ dimensions.

\section{Physical meaning of difficulties in $8 n, 1+8 n$ and $2+8 n$ dimensions}

Let us go back to table 1 in section 1 in which results of our analysis are summarized. Entries with the cross $x$ in this table are those for which we found difficulties. In what follows, we give an argument that these difficulties have an intrinsic physical meaning.

Let us start our discussion by recalling following facts. In $d$ dimensional Minkowski spacetime, when $d=0,4 \bmod 8$, the Majorana fermion and the Weyl fermion in a real representation are equivalent. This means that one can convert the Majorana fermion into the real Weyl fermion by multiplying the projection operator $(1-\gamma) / 2$. This operation is invertible because one can reproduce the original Majorana fermion by further multiplying $1+\mathcal{C}$, where $\mathcal{C}$ is the charge conjugation operation. For this, relations $\mathcal{C}^{2}=1$ and $\{\mathcal{C}, \gamma\}=0$ (the charge conjugation flips the chirality) holding in these dimensions are crucial. Similarly, one can convert the real Weyl fermion into the Majorana fermion by multiplying $1+\mathcal{C}$; this operation is again invertible. As expected from this equivalence, Weyl fermions in a real representation are free from the local gauge anomaly in $8 n$ and $4+8 n$ dimensions.

Now in $4+8 n$ dimensions, this equivalence between real Weyl fermions and Majorana fermions holds in euclidean lattice gauge theory. Furthermore, these fermions are free from any pathologies even in a non-perturbative level (this is consistent with the fact that there is no global gauge anomaly for real representations in $4+8 n$ dimensions [39]). These facts can be shown by repeating the argument of ref. [21].

How is the situation in $8 n$ dimensions? It is natural to expect that any sensible non-perturbative formulation preserves the above equivalence between Majorana and real Weyl, because it is a basic property of the target theory. Then we find a trouble. In $8 n$ dimensions, it has been known that Weyl fermions in a real representation generally exhibit the global gauge anomaly ( pseudo-real representations, on the other hand, are free from the global gauge anomaly [39]). For example, in 8 dimensions, the Weyl fermion belonging to the 20' representation of the gauge-group SU(4) exhibits the global gauge anomaly $\left(\pi_{8}[\mathrm{SU}(4)]=Z_{4 !}\right)$. Similarly, in 16 dimensions, the Weyl fermion in the $\mathbf{7 0}$ 
representation of $\mathrm{SU}(8)$ is anomalous $\left(\pi_{16}[\mathrm{SU}(8)]=Z_{8 !}\right)$ [40]. So if it was possible to obtain a simple formulation of the Majorana fermion in $8 n$ dimensions such as eq. (2.10) (note that this formulation does not refer to a particular real representation), then either the equivalence with real Weyl fermions is lost or the global gauge anomaly is not reproduced. From this argument, we regard a difficulty of the Majorana decomposition in $8 n$ dimensions is a reflection of the global gauge anomaly.

Next, we consider $d=1+8 n$ cases. We note that the Majorana fermion in $1+8 n$ dimensions, after the dimensional reduction, becomes the Majorana fermion in $8 n$ dimensions. This is expected even on the lattice; if it is possible to construct a lattice action for the Majorana fermion in $1+8 n$ dimensions, then the dimensional reduction explained in section 5.2 will provide a lattice action of the Majorana fermion in $8 n$ dimensions. However, we argued above that such a simple action of the Majorana fermion in $8 n$ dimensions is quite unlikely. So, $d=1+8 n$ cases are also expected to be difficult. Similarly, the dimensional reduction of the Majorana-Weyl fermion in $2+8 n$ dimensions will give rise to the Majorana fermion in $1+8 n$ dimensions. So again we expect a difficulty. ${ }^{13}$

In this way, through a ladder of dimensional reductions, three cases, the MajoranaWeyl fermion in $2+8 n$ dimensions, the Majorana fermion in $1+8 n$ dimensions and the Majorana fermion in $8 n$ dimensions, are related. Then by assuming an equivalence of the Majorana fermion and the Weyl fermion in a real representation in $8 n$ dimensions, difficulties we found in this paper are related to the global gauge anomaly. This argument indicates that a lattice formulation of these fermions is extremely difficult to find. Since the gaugino field of the $N=1$ super Yang-Mills theory in these dimensions is precisely represented by these fermions, ${ }^{14}$ our argument suggests also that a lattice formulation of the $N=1$ super Yang-Mills theory in these dimensions is extremely difficult to find for this reason (besides other well-known reasons for supersymmetric theories on the lattice). ${ }^{15}$

Finally, here is a list of further study. It will be possible to generalize the argument of ref. [42] to $8 n$ dimensions and study the global gauge anomaly of real Weyl fermions in these dimensions. (For pseudo-real Weyl fermions, an argument similar to that of ref. 221] will be applied.) It will also be interesting to investigate a possible non-perturbative pathology of Majorana fermions in $8 n$ and $1+8 n$ dimensions by using lattice gauge theory, although we do not know of any pathologies in the latter odd dimensions in continuum theory. ${ }^{16}$ An analysis of Majorana-Weyl fermions in $2+8 n$ dimensions will be difficult because a reality of the gauge-group representation does not imply an absence of the local gauge

\footnotetext{
${ }^{13}$ It is possible that Majorana-Weyl fermions in $2+8 n$ dimensions themselves suffer from the global gauge anomaly, although we do not know of an example. Real Weyl fermions in $2+8 n$ dimensions are known to be free from the global gauge anomaly, when being free from the local gauge anomaly 441.

${ }^{14}$ Here, we define a number of supercharges $N$ by taking the smallest irreducible spinor representation as a unit. In this convention, the supercharge of the $N=1$ super Yang-Mills theory in $2+8 n$ dimensions is a single Majorana-Weyl fermion.

${ }^{15}$ A possible way out for the $N=1$ super Yang-Mills theory in $8 n$ dimensions is to formulate the gaugino field as the lattice Weyl fermion. For this case, however, the transformation parameter of the supersymmetry transformation will be a subject of the constraint (3.4) that depends on the gauge field.

${ }^{16}$ It is interesting to note that the Majorana fermion in $1+8 n$ dimensions suffers from the global gravitational anomaly [43.
} 
anomaly. It might also be possible to establish a no-go theorem for lattice Majorana and Majorana-Weyl fermions in these dimensions, that is analogous to the Nielsen-Ninomiya theorem [25]. ${ }^{17}$ We hope to come back some of these problems in the near future.

\section{Acknowledgments}

H.S. would like to thank Takanori Fujiwara, Yoshio Kikukawa and Kiyoshi Okuyama for helpful discussions.

\section{A. Representation of the Clifford algebra in $d$ dimensional euclidean space}

Here we summarize our conventions for gamma matrices. Our gamma matrices are hermitiean and satisfy the Clifford algebra

$$
\left\{\gamma_{\mu}, \gamma_{\nu}\right\}=2 \delta_{\mu \nu}, \quad \mu, \nu=0,1, \ldots, d-1
$$

We start with cases of even $(d=2 k)$ dimensional space. In even dimensions, the chiral matrix can be defined by

$$
\gamma=i^{-k} \gamma_{0} \gamma_{1} \cdots \gamma_{2 k-1}
$$

such that

$$
\left\{\gamma, \gamma_{\mu}\right\}=0, \quad \gamma^{\dagger}=\gamma, \quad \gamma^{2}=1
$$

Since $\pm \gamma_{\mu}^{*}$ obey the Clifford algebra identical to eq. (A.1), $\gamma_{\mu}$ and $\pm \gamma_{\mu}^{*}$ are related by similarity transformations

$$
B_{1} \gamma_{\mu} B_{1}^{-1}=(-1)^{k} \gamma_{\mu}^{*}=(-1)^{k} \gamma_{\mu}^{T}, \quad B_{2} \gamma_{\mu} B_{2}^{-1}=(-1)^{k+1} \gamma_{\mu}^{*}=(-1)^{k+1} \gamma_{\mu}^{T}
$$

One can choose the phase of these matrices such that

$$
B_{1}^{-1}=B_{1}^{\dagger}, \quad B_{2}^{-1}=B_{2}^{\dagger}
$$

These $B_{1}$ and $B_{2}$ are referred to as charge conjugation matrices in this paper. Note that

$$
B_{1} \gamma B_{1}^{-1}=B_{2} \gamma B_{2}^{-1}=(-1)^{k} \gamma^{*}=(-1)^{k} \gamma^{T} .
$$

${ }^{17}$ In this respect, it is instructive to see what happens if one adopts the action

$$
S=\int \mathrm{d}^{d} x \frac{1}{2} \chi^{T}(x) B_{1} D_{\mathrm{w}} \chi(x),
$$

for $d=8 n$ or $d=1+8 n$, despite the fact that $\left(B_{1} D_{\mathrm{W}}\right)^{T} \neq-B_{1} D_{\mathrm{W}}$. In this case, one has

$$
\begin{aligned}
S & =\int \mathrm{d}^{d} x \frac{1}{4} \chi^{T}(x)\left[B_{1} D_{\mathrm{W}}-\left(B_{1} D_{\mathrm{W}}\right)^{T}\right] \chi(x) \\
& =\int \mathrm{d}^{d} x \frac{1}{4} \chi^{T}(x) B_{1} \gamma_{\mu}\left(\nabla_{\mu}+\nabla_{\mu}^{*}\right) \chi(x),
\end{aligned}
$$

and the species doubling occurs. A similar conclusion is obtained for the overlap-Dirac operator. 
We adopt a representation of gamma matrices such that ${ }^{18}$

$$
\begin{aligned}
& \gamma_{0}, \gamma_{2}, \ldots, \gamma_{2 k-2}: \text { real } \\
& \gamma_{1}, \gamma_{3}, \ldots, \gamma_{2 k-1}: \text { purely imaginary }
\end{aligned}
$$

In this representation, the chiral matrix is real, $\gamma^{*}=\gamma^{T}=\gamma$. Charge conjugation matrices in our representation are then given by

$$
B_{1}=\gamma_{1} \gamma_{3} \ldots \gamma_{2 k-1}, \quad B_{2}=\gamma B_{1}
$$

With a help of these explicit forms, we find

$$
\begin{aligned}
B_{1}^{T} & =(-1)^{k(k+1) / 2} B_{1}, & B_{2}^{T} & =(-1)^{k(k-1) / 2} B_{2}, \\
B_{1}^{*} B_{1} & =(-1)^{k(k+1) / 2}, & B_{2}^{*} B_{2} & =(-1)^{k(k-1) / 2},
\end{aligned}
$$

but actually these relations hold irrespective of the representation. ${ }^{19}$ As relations particularly hold in our representation, we have

$$
\begin{aligned}
& B_{1}^{*}=(-1)^{k} B_{1}, \quad B_{2}^{*}=(-1)^{k} B_{2}, \\
& B_{1}^{2}=(-1)^{k(k-1) / 2}, \quad B_{2}^{2}=(-1)^{k(k+1) / 2} .
\end{aligned}
$$

For odd $(d=2 k+1)$ dimensional space, gamma matrices are obtained from those of $2 k$ dimensions by

$$
\gamma_{\mu}, \quad \mu=0,1, \ldots, 2 k-1 \quad \gamma_{2 k}=\gamma
$$

From eqs. (A.4) and (A.6), one sees that only $B_{1}$ is the charge conjugation matrix such that

$$
B_{1} \gamma_{\mu} B_{1}^{-1}=(-1)^{k} \gamma_{\mu}^{*}=(-1)^{k} \gamma_{\mu}^{T}, \quad \mu=0,1, \ldots, 2 k
$$

\section{B. Charge conjugation properties of lattice Dirac operators}

The lattice covariant differences are defined by

$$
\begin{aligned}
& \nabla_{\mu} \psi(x)=\frac{1}{a}\{R[U(x, \mu)] \psi(x+a \hat{\mu})-\psi(x)\}, \\
& \nabla_{\mu}^{*} \psi(x)=\frac{1}{a}\left\{\psi(x)-R[U(x-a \hat{\mu}, \mu)]^{-1} \psi(x-a \hat{\mu})\right\},
\end{aligned}
$$

\footnotetext{
${ }^{18}$ For example, we can take

$$
\begin{aligned}
& \gamma_{0}=\sigma_{1} \otimes \sigma_{3} \otimes \sigma_{3} \otimes \cdots \otimes \sigma_{3} \otimes \sigma_{3}, \quad \gamma_{1}=\sigma_{2} \otimes \sigma_{3} \otimes \sigma_{3} \otimes \cdots \otimes \sigma_{3} \otimes \sigma_{3}, \\
& \gamma_{2}=1 \otimes \sigma_{1} \otimes \sigma_{3} \otimes \cdots \otimes \sigma_{3} \otimes \sigma_{3}, \quad \gamma_{3}=1 \otimes \sigma_{2} \otimes \sigma_{3} \otimes \cdots \otimes \sigma_{3} \otimes \sigma_{3}, \\
& \gamma_{2 k-2}=1 \otimes 1 \otimes 1 \otimes \cdots \otimes 1 \otimes \sigma_{1}, \quad \gamma_{2 k-1}=1 \otimes 1 \otimes 1 \otimes \cdots \otimes 1 \otimes \sigma_{2},
\end{aligned}
$$

and

$$
\gamma=\sigma_{3} \otimes \cdots \otimes \sigma_{3}
$$

${ }^{19}$ Under a change of the representation, $\gamma_{\mu} \rightarrow U^{-1} \gamma_{\mu} U$, where $U$ is a unitary matrix, a charge conjugation matrix $B$ changes as $B \rightarrow U^{T} B U$.
} 
where $U(x, \mu)$ denotes a link variable and $R$ is the gauge-group representation to which the fermion belongs; $\hat{\mu}$ is the unit vector in the direction $\mu$. We assume that $R$ is a real representation in what follows. The Wilson-Dirac operator is defined by

$$
D_{\mathrm{W}}=\frac{1}{2}\left[\gamma_{\mu}\left(\nabla_{\mu}^{*}+\nabla_{\mu}\right)-a \nabla_{\mu}^{*} \nabla_{\mu}\right]
$$

From the relation $\nabla_{\mu}^{T}=-\nabla_{\mu}^{*}$ and formulas in appendix $\mathrm{A}$, we find

$$
D_{\mathrm{W}}^{T}= \begin{cases}B_{2} D_{\mathrm{W}} B_{2}^{-1}, \quad B_{2}^{T}=+B_{2}, & \text { for } d=8 n, \\ \text { no simple relation } & \text { for } d=1+8 n, \\ B_{1} D_{\mathrm{W}} B_{1}^{-1}, \quad B_{1}^{T}=-B_{1}, & \text { for } d=2+8 n, \\ B_{1} D_{\mathrm{W}} B_{1}^{-1}, \quad B_{1}^{T}=-B_{1}, & \text { for } d=3+8 n, \\ B_{2} D_{\mathrm{W}} B_{2}^{-1}, \quad B_{2}^{T}=-B_{2}, & \text { for } d=4+8 n,\end{cases}
$$

and

$$
D_{\mathrm{W}}^{*}= \begin{cases}B_{1} D_{\mathrm{W}} B_{1}^{-1}, \quad B_{1}^{T}=+B_{1}, & \text { for } d=8 n, \\ B_{1} D_{\mathrm{W}} B_{1}^{-1}, \quad B_{1}^{T}=+B_{1}, & \text { for } d=1+8 n, \\ B_{2} D_{\mathrm{W}} B_{2}^{-1}, \quad B_{2}^{T}=+B_{2}, & \text { for } d=2+8 n, \\ \text { no simple relation, } & \text { for } d=3+8 n, \\ B_{1} D_{\mathrm{W}} B_{1}^{-1}, \quad B_{1}^{T}=-B_{1}, & \text { for } d=4+8 n .\end{cases}
$$

The overlap-Dirac operator is defined by

$$
D=\frac{1}{a}\left[1-A\left(A^{\dagger} A\right)^{-1 / 2}\right], \quad A=\frac{1}{a}-D_{\mathrm{W}},
$$

from the Wilson-Dirac operator $D_{\mathrm{W}}$. For this operator, we have

$$
D^{T}= \begin{cases}B_{2} D B_{2}^{-1}, \quad B_{2}^{T}=+B_{2}, & \text { for } d=8 n \\ \text { no simple relation } & \text { for } d=1+8 n \\ B_{1} D B_{1}^{-1}, \quad B_{1}^{T}=-B_{1}, & \text { for } d=2+8 n \\ \text { no simple relation } & \text { for } d=3+8 n \\ B_{2} D B_{2}^{-1}, \quad B_{2}^{T}=-B_{2}, & \text { for } d=4+8 n .\end{cases}
$$

\section{Positivity of the Majorana pfaffian in $2+8 n$ and $4+8 n$ dimensions}

In this appendix, we demonstrate that the pfaffian (2.10) for $d=2+8 n$ and for $d=4+8 n$ is a non-negative function of a gauge-field configuration, up to a proportionality constant. To show this, we use the eigenfunctions of the hermitean operator $H(2.13)$ :

$$
H \varphi_{n}(x)=\lambda_{n} \varphi_{n}(x), \quad\left(\varphi_{m}, \varphi_{n}\right)=\delta_{m, n} .
$$

Also we recall the lattice index theorem [3]

$$
\operatorname{Tr} \Gamma=n_{+}-n_{-},
$$

where the operator $\Gamma$ is defined by eq. (2.14); $n_{+}$and $n_{-}$denote a number of zero modes of positive and negative chiralities, respectively. This relation can be shown by noting that the mode $\Gamma \varphi_{n}$ is orthogonal to $\varphi_{n}$ for $\lambda_{n} \neq 0$, because it has an opposite-sign eigenvalue $-\lambda_{n}$ 
due to eq. (2.15). One can also show the chirality sum-rule 44

$$
\operatorname{Tr} \gamma=n_{+}-n_{-}+N_{+}-N_{-}=0
$$

where $N_{+}$and $N_{-}$denote a number of modes such that $H \varphi_{n}=+(2 / a) \varphi_{n}$ and $H \varphi_{n}=$ $-(2 / a) \varphi_{n}$, respectively.

We start with the case of $d=2+8 n$. First we note that for $d=2+8 n$, the transpose of $\Gamma$ is given by $\Gamma^{T}=-B_{2} \Gamma B_{2}^{-1}$ and the index vanishes

$$
n_{+}-n_{-}=\operatorname{Tr} \Gamma=-\operatorname{Tr} \Gamma=0 .
$$

The Dirac fermion in a real representation is thus insensitive to a difference of topological sectors; all possible zero modes are not topological but accidental. Having this fact in mind, we evaluate the functional integral

$$
\langle 1\rangle_{\mathrm{M}}=\int \prod_{x} \mathrm{~d} \chi(x) \exp \left[-a^{d} \sum_{x} \frac{1}{2} \chi^{T}(x) B_{1} D \chi(x)\right],
$$

by expanding the field $\chi(x)$ by a complete system of functions. We first take eigenfunctions $\varphi_{n}$ in eq. (C.1) with positive eigenvalues $\lambda_{n}>0$ only. From these functions, we can construct eigenfunctions with negative eigenvalues by $B_{2}^{-1} \varphi_{n}^{*}$,

$$
H B_{2}^{-1} \varphi_{n}^{*}(x)=-\lambda_{n} B_{2}^{-1} \varphi_{n}^{*}(x), \quad \lambda_{n}>0 .
$$

Since $B_{2}^{*} B_{2}=1,\left\{\varphi_{n}, B_{2}^{-1} \varphi_{n}^{*}\right\}$ form a complete system. We thus expand the field in terms of these eigenfunctions

$$
\chi(x)=\sum_{\lambda_{n}>0}\left[\varphi_{n}(x) c_{n}+B_{2}^{-1} \varphi_{n}^{*}(x) b_{n}\right]
$$

Then by noting $B_{1} D=-B_{2} H$ and $\left(\varphi_{m}, B_{2}^{-1} \varphi_{n}^{*}\right)=0$, the action becomes

$$
-a^{d} \sum_{x} \frac{1}{2} \chi^{T}(x) B_{1} D \chi(x)=\sum_{\lambda_{n}>0} \lambda_{n} b_{n} c_{n} .
$$

For the integration measure, we have $\prod_{x} \mathrm{~d} \chi(x)=\operatorname{det}^{-1} U \prod_{n=1}^{(1 / 2) \operatorname{Tr} 1} \mathrm{~d} c_{n} \prod_{n=1}^{(1 / 2) \operatorname{Tr} 1} \mathrm{~d} b_{n}$ under the change of variables, where the matrix $U$ is defined by

$$
U_{x, n}=\left[\varphi_{n}(x), B_{2}^{-1} \varphi_{n}^{*}(x)\right]
$$

For this matrix, one has the relation

$$
\sum_{x, l} U_{m, x}^{T} B_{2} U_{x, l}\left[\begin{array}{cc}
0 & \delta_{l, n} \\
\delta_{l, n} & 0
\end{array}\right]=\left[\begin{array}{cc}
\delta_{m, n} & 0 \\
0 & \delta_{m, n}
\end{array}\right],
$$

from the orthonormality of $\varphi_{n}$ and thus

$$
\operatorname{det}^{-2} U=(-1)^{[(1 / 2) \operatorname{Tr} 1]^{2}} \operatorname{det} B_{2} .
$$


In this way, we have

$$
\langle 1\rangle_{\mathrm{M}}= \pm e^{i \alpha / 2} \prod_{\lambda_{n}>0} \lambda_{n}, \quad e^{i \alpha}=(-1)^{[(1 / 2) \operatorname{Tr} 1]^{2}} \operatorname{det} B_{2} .
$$

The over-all \pm sign in eq. (C.12) has to be chosen such that $\langle 1\rangle_{M}$ changes smoothly when a certain eigenvalue crosses zero (otherwise there may be a cusp; recall that eigenvalues come in pair as $\lambda_{n}$ and $\left.-\lambda_{n}\right)$, because we know that $\langle 1\rangle_{\mathrm{M}}=\operatorname{Pf}\left(B_{1} D\right)$ is definitely a smooth function of a gauge-field configuration.

Fortunately, it turns out that this artificial sign flip is not necessary. ${ }^{20}$ To see this, we note the fact that eigenvalues $\lambda_{n} \neq 2 / a$ have a double degeneracy (this follows from a good chiral property of the overlap-Dirac operator). Namely, the mode

$$
\phi_{n}(x)=\frac{1}{\sqrt{1-a^{2} \lambda_{n}^{2} / 4}} \Gamma B_{2}^{-1} \varphi_{n}^{*}(x),
$$

has an identical eigenvalue $\lambda_{n}$ to $\varphi_{n}$. This mode $\phi_{n}$ is linearly independent from $\varphi_{n}$ because $\left(\varphi_{n}, \phi_{n}\right)=0$ holds due to $\left(\Gamma B_{2}^{-1}\right)^{T}=-\Gamma B_{2}^{-1}$. From this fact, we have

$$
\langle 1\rangle_{\mathrm{M}}=e^{i \alpha / 2}(2 / a)^{N_{+}} \prod_{0<\lambda_{n} \neq 2 / a} \lambda_{n}^{2},
$$

where we have taken the + sign in eq. (C.12) for all gauge-field configurations, because then the expression becomes a smooth function. The product $\prod_{\lambda_{n}}$ in this expression is understood to be omitting the above double degeneracy, i.e., one factor $\lambda_{n}^{2}$ for each pair of $\varphi_{n}$ and $\phi_{n}$. This expression proves that $\langle 1\rangle_{\mathrm{M}}$ is, up to a proportionality constant, a non-negative function of a gauge-field configuration.

Next, we consider the case of $d=4+8 n$. The following analysis is basically identical to that of ref. 21]. For this case, the index $n_{+}-n_{-}$does not vanish in general and there may exist topological zero modes. To avoid a complication associated to these zero modes, it is convenient to introduce a mass term [21]. Thus, we consider

$$
\langle 1\rangle_{\mathrm{M}}=\int \prod_{x} \mathrm{~d} \chi(x) \exp \left\{-a^{d} \sum_{x}\left[\frac{1}{2} \chi^{T}(x) B_{2} D \chi(x)+\frac{1}{2} i m \chi^{T}(x) B_{1} \chi(x)\right]\right\} .
$$

For the present case, we use all the eigenfunctions $\varphi_{n}$ in eq. (C.1) to expand the field. However, there is a double degeneracy, namely, the mode

$$
\phi_{n}(x)=B_{1}^{-1} \varphi_{n}^{*}(x),
$$

has the eigenvalue identical to that of the mode $\varphi_{n}$. This is linearly independent from $\varphi_{n}$, because $\left(\varphi_{n}, \phi_{n}\right)=0$ holds (note $B_{1}^{T}=-B_{1}$ ). In particular, $n_{ \pm}$and $N_{ \pm}$are even numbers. Noting these facts, we expand the field as

$$
\chi(x)=\sum_{\lambda_{n}}\left[\varphi_{n}(x) c_{n}+B_{1}^{-1} \varphi_{n}^{*}(x) b_{n}\right]
$$

\footnotetext{
${ }^{20}$ This is not the case for the Wilson-Dirac operator. One has to flip the \pm sign in eq. (C.12) at each time when an eigenvalue crosses zero. As the result, the pfaffian is not necessarily non-negative.
} 
Then by using $B_{2} D=B_{1} H$, the action becomes

$$
-a^{d} \sum_{x}\left[\frac{1}{2} \chi^{T}(x) B_{2} D \chi(x)+\frac{1}{2} i m \chi^{T}(x) B_{1} \chi(x)\right]=\sum_{\lambda_{n}}\left(\lambda_{n}+i m\right) b_{n} c_{n} .
$$

For the integration measure, we have $\prod_{x} \mathrm{~d} \chi(x)=\operatorname{det}^{-1} V \prod_{n=1}^{(1 / 2) \operatorname{Tr} 1} \mathrm{~d} c_{n} \prod_{n=1}^{(1 / 2) \operatorname{Tr} 1} \mathrm{~d} b_{n}$, where the matrix $V$ is defined by

$$
V_{x, n}=\left[\varphi_{n}(x), B_{1}^{-1} \varphi_{n}^{*}(x)\right],
$$

and, corresponding to eq. $(\overline{\mathrm{C} .10})$,

$$
\sum_{x, l} V_{m, x}^{T} B_{1} V_{x, l}\left[\begin{array}{cc}
0 & -\delta_{l, n} \\
\delta_{l, n} & 0
\end{array}\right]=\left[\begin{array}{cc}
\delta_{m, n} & 0 \\
0 & \delta_{m, n}
\end{array}\right],
$$

and

$$
\operatorname{det}^{-2} V=(-1)^{[(1 / 2) \operatorname{Tr} 1]^{2}}(-1)^{(1 / 2) \operatorname{Tr} 1} \operatorname{det} B_{2} .
$$

After the integration over $c_{n}$ and $b_{n}$, we thus have

$$
\langle 1\rangle_{\mathrm{M}}= \pm e^{i \beta / 2}(-1)^{(1 / 4) \operatorname{Tr} 1} \prod_{\lambda_{n}}\left(\lambda_{n}+i m\right), \quad e^{i \beta}=(-1)^{[(1 / 2) \operatorname{Tr} 1]^{2}} \operatorname{det} B_{1},
$$

where the product $\prod_{\lambda_{n}}$ is understood to be omitting the double degeneracy, i.e., one factor $\lambda_{n}+i m$ for each pair of $\varphi_{n}$ and $\phi_{n}$. At this point, we further note that eigenvalues $\lambda_{n} \neq 0$ and $\lambda_{n} \neq \pm 2 / a$ appear in pair as $\lambda_{n}$ and $-\lambda_{n}$, because

$$
\frac{1}{\sqrt{1-a^{2} \lambda_{n}^{2} / 4}} \Gamma \varphi_{n}(x)
$$

has the eigenvalue $-\lambda_{n}$. From these facts, we have

$$
\begin{aligned}
\langle 1\rangle_{\mathrm{M}}= & e^{i \beta / 2}(-1)^{(1 / 4) \operatorname{Tr} 1}(i m)^{\left(n_{+}+n_{-}\right) / 2}(2 / a+i m)^{N_{+} / 2}(-2 / a+i m)^{N_{-} / 2} \times \\
& \times \prod_{0<\lambda_{n} \neq 2 / a}\left(-\lambda_{n}+i m\right)\left(\lambda_{n}+i m\right),
\end{aligned}
$$

where we have taken the + sign in eq. (C.22) for all configurations, because then the expression becomes a smooth function. Then from a counting of a number of eigenfunctions, one finds that a number of terms in the last product is $\left(\operatorname{Tr} 1-N_{+}-N_{-}-n_{+}-n_{-}\right) / 4$. Therefore, we finally have

$$
\begin{aligned}
\langle 1\rangle_{\mathrm{M}}= & e^{i \beta / 2}(-1)^{\left(n_{+}-n_{-}\right) / 4}(2 / a-i m)^{\left(n_{+}-n_{-}\right) / 2} \times \\
& \times m^{\left(n_{+}+n_{-}\right) / 2}\left(4 / a^{2}+m^{2}\right)^{N_{+} / 2} \prod_{0<\lambda_{n} \neq 2 / a}\left(\lambda_{n}^{2}+m^{2}\right),
\end{aligned}
$$

where a use of the chirality sum-rule $(\overline{C .3})$ has been made. It is interesting to note that the prefactor $(-1)^{\left(n_{+}-n_{-}\right) / 4}(2 / a-i m)^{\left(n_{+}-n_{-}\right) / 2}$ in this expression depends only on a topological sector concerned through the index $n_{+}-n_{-}$. Assuming that there is no zero modes, this expression reduces to

$$
\langle 1\rangle_{\mathrm{M}}=e^{i \beta / 2}(2 / a)^{N_{+}} \prod_{0<\lambda_{n} \neq 2 / a} \lambda_{n}^{2},
$$

in the massless limit. This proves that the pfaffian is, up to a proportionality constant, a non-negative function of a gauge-field configuration. 


\section{References}

[1] K.G. Wilson, Quarks and strings on a lattice, in New phenomena in subnuclear physics. Part A, A. Zichichi ed., Plenum Press, New York, 1977, p. 69, proceedings of the first half of the 1975 International School of Subnuclear Physics, Erice, Sicily, July 11-August 1, 1975.

[2] H. Neuberger, Exactly massless quarks on the lattice, Phys. Lett. B 417 (1998) 141 hep-lat/9707022]; More about exactly massless quarks on the lattice, Phys. Lett. B 427 (1998) 353 hep-lat/9801031.

[3] P. Hasenfratz, Prospects for perfect actions, Nucl. Phys. 63 (Proc. Suppl.) (1998) 53 [hep-lat/9709110]; Lattice QCD without tuning, mixing and current renormalization, Nucl. Phys. B 525 (1998) 401 hep-lat/9802007;

P. Hasenfratz, V. Laliena and F. Niedermayer, The index theorem in QCD with a finite cut-off, Phys. Lett. B 427 (1998) 125 hep-lat/9801021].

[4] H. Nicolai, A possible constructive approach to (super- $\left.\phi^{3}\right)$ in four-dimensions. (I). Euclidean formulation of the model, Nucl. Phys. B 140 (1978) 294; A possible constructive approach to super- $\phi^{3}$ in four-dimensions. (II). Regularization of the model, Nucl. Phys. B 156 (1979) 157; A possible constructive approach to (super- $\phi^{3}$ ) in four-dimensions. (III). On the normalization of schwinger functions, Nucl. Phys. B 156 (1979) 177.

[5] P. van Nieuwenhuizen and A. Waldron, On euclidean spinors and Wick rotations, Phys. Lett. B 389 (1996) 29 hep-th/9608174, and references therein.

[6] K. Fujikawa and M. Ishibashi, Lattice chiral symmetry and the Wess-Zumino model, Nucl. Phys. B 622 (2002) 115 hep-th/0109156; Lattice chiral symmetry, Yukawa couplings and the Majorana condition, Phys. Lett. B 528 (2002) 295 hep-lat/0112050.

[7] K. Fujikawa, M. Ishibashi and H. Suzuki, Ginsparg-Wilson operators and a no-go theorem, Phys. Lett. B 538 (2002) 197 hep-lat/0202017.

[8] P. Hasenfratz, Lattice 2001: reflections, Nucl. Phys. 106 (Proc. Suppl.) (2002) 159 hep-lat/0111023.

[9] K. Fujikawa, M. Ishibashi and H. Suzuki, CP breaking in lattice chiral gauge theories, J. High Energy Phys. 04 (2002) 046 hep-lat/0203016;

K. Fujikawa and H. Suzuki, Domain wall fermion and CP symmetry breaking, Phys. Rev. D 67 (2003) 034506 hep-lat/0210013.

[10] Y. Kikukawa and H. Neuberger, Overlap in odd dimensions, Nucl. Phys. B 513 (1998) 735 hep-lat/9707016.

[11] R. Narayanan and H. Neuberger, Infinitely many regulator fields for chiral fermions, Phys. Lett. B 302 (1993) 62 hep-lat/9212019; Chiral determinant as an overlap of two vacua, Nucl. Phys. B 412 (1994) 574] [hep-lat/9307006]; Chiral fermions on the lattice, Phys. Rev. Lett. 71 (1993) 3251 [hep-lat/9308011]; A construction of lattice chiral gauge theories, Nucl. Phys. B 443 (1995) 305 hep-th/9411108;

S. Randjbar-Daemi and J. Strathdee, On the overlap formulation of chiral gauge theory, Phys. Lett. B 348 (1995) 543 [hep-th/9412165]; Chiral fermions on the lattice, Nucl. Phys. B 443 (1995) 386 [hep-lat/9501027]; On the overlap prescription for lattice regularization of chiral fermions, Nucl. Phys. B 466 (1996) 335 hep-th/9512112; Consistent and covariant anomalies in the overlap formulation of chiral gauge theories, Phys. Lett. B 402 (1997) 134 hep-th/9703092. 
[12] P.Y. Huet, R. Narayanan and H. Neuberger, Overlap formulation of Majorana-Weyl fermions, Phys. Lett. B 380 (1996) 291 hep-th/9602176;

R. Narayanan and H. Neuberger, Overlap for Majorana-Weyl fermions, Nucl. Phys. 53 (Proc. Suppl.) (1997) 658 hep-lat/9607080].

[13] N. Maru and J. Nishimura, Lattice formulation of supersymmetric Yang-Mills theories without fine-tuning, Int. J. Mod. Phys. A 13 (1998) 2841 hep-th/9705152.

[14] N. Kitsunezaki and J. Nishimura, Unitary IIB matrix model and the dynamical generation of the space time, Nucl. Phys. B 526 (1998) 351 hep-th/9707162.

[15] E. Witten, An SU(2) anomaly, Phys. Lett. B 117 (1982) 324.

[16] S. Elitzur and V.P. Nair, Nonperturbative anomalies in higher dimensions, Nucl. Phys. B 243 (1984) 205.

[17] P.H. Ginsparg and K.G. Wilson, A remnant of chiral symmetry on the lattice, Phys. Rev. D 25 (1982) 2649.

[18] M. Lüscher, Exact chiral symmetry on the lattice and the Ginsparg-Wilson relation, Phys. Lett. B 428 (1998) 342 hep-lat/9802011.

[19] R. Narayanan, Ginsparg-Wilson relation and the overlap formula, Phys. Rev. D 58 (1998) 097501 hep-lat/9802018];

F. Niedermayer, Exact chiral symmetry, topological charge and related topics, Nucl. Phys. 73 (Proc. Suppl.) (1999) 105 hep-lat/9810026.

[20] P. Hernández, K. Jansen and M. Lüscher, Locality properties of Neuberger's lattice Dirac operator, Nucl. Phys. B 552 (1999) 363 hep-lat/9808010;

H. Neuberger, Bounds on the Wilson Dirac operator, Phys. Rev. D 61 (2000) 085015 hep-lat/9911004.

[21] H. Suzuki, Real representation in chiral gauge theories on the lattice, J. High Energy Phys. 10 (2000) 039 hep-lat/0009036.

[22] H. Neuberger, Vector like gauge theories with almost massless fermions on the lattice, Phys. Rev. D 57 (1998) 5417 hep-lat/9710089.

[23] D.B. Kaplan and M. Schmaltz, Supersymmetric Yang-Mills theories from domain wall fermions, Chin. J. Phys. 38 (2000) 543 hep-lat/0002030.

[24] For a review see A. Feo, Supersymmetry on the lattice, Nucl. Phys. 119 (Proc. Suppl.) (2003) 198 hep-lat/0210015, and references therein.

[25] H.B. Nielsen and M. Ninomiya, Absence of neutrinos on a lattice, 1. Proof by homotopy theory, Nucl. Phys. B 185 (1981) 20, erratum ibid. B 195 (1982) 541; No go theorem for regularizing chiral fermions, Phys. Lett. B 105 (1981) 219;

D. Friedan, A proof of the Nielsen-Ninomiya theorem, Commun. Math. Phys. 85 (1982) 481.

[26] B. Bunk, M. Della Morte, K. Jansen and F. Knechtli, Locality with staggered fermions, hep-lat/0403022.

[27] D.B. Kaplan, A method for simulating chiral fermions on the lattice, Phys. Lett. B 288 (1992) 342 hep-lat/9206013.

[28] M. Lüscher, Abelian chiral gauge theories on the lattice with exact gauge invariance, Nucl. Phys. B 549 (1999) 295 hep-lat/9811032. 
[29] M. Lüscher, Weyl fermions on the lattice and the non-abelian gauge anomaly, Nucl. Phys. B $\mathbf{5 6 8}$ (2000) 162 hep-lat/9904009.

[30] H. Suzuki, Anomaly cancellation condition in lattice gauge theory, Nucl. Phys. B 585 (2000) 471 hep-lat/0002009;

H. Igarashi, K. Okuyama and H. Suzuki, Errata and addenda to "Anomaly cancellation condition in lattice gauge theory", hep-lat/0012018.

[31] Y. Kikukawa and Y. Nakayama, Gauge anomaly cancellations in $\mathrm{SU}(2)_{L} \times \mathrm{U}(1)_{Y}$ electroweak theory on the lattice, Nucl. Phys. B 597 (2001) 519 hep-lat/0005015.

[32] M. Lüscher, Lattice regularization of chiral gauge theories to all orders of perturbation theory, J. High Energy Phys. 06 (2000) 028 hep-lat/0006014.

[33] Y. Kikukawa, Domain wall fermion and chiral gauge theories on the lattice with exact gauge invariance, Phys. Rev. D 65 (2002) 074504 hep-lat/0105032.

[34] D. Kadoh, Y. Kikukawa and Y. Nakayama, Solving the local cohomology problem in U(1) chiral gauge theories within a finite lattice, hep-lat/0309022,

D. Kadoh and Y. Kikukawa, A numerical solution to the local cohomology problem in $\mathrm{U}(1)$ chiral gauge theories, hep-lat/0401025.

[35] M. Golterman and Y. Shamir, SU(N) chiral gauge theories on the lattice, hep-lat/0404011, and references therein.

[36] A.N. Redlich, Gauge noninvariance and parity nonconservation of three- dimensional fermions, Phys. Rev. Lett. 52 (1984) 18; Parity violation and gauge noninvariance of the effective gauge field action in three-dimensions, Phys. Rev. D 29 (1984) 2366;

A.J. Niemi and G.W. Semenoff, Axial anomaly induced fermion fractionization and effective gauge theory actions in odd dimensional space-times, Phys. Rev. Lett. 51 (1983) 2077.

[37] H. So, Induced Chern-Simons class with lattice fermions, Prog. Theor. Phys. 73 (1985) 528; Induced topological invariants by lattice fermions in odd dimensions, Prog. Theor. Phys. 74 (1985) 585;

A. Coste and M. Lüscher, Parity anomaly and fermion boson transmutation in three-dimensional lattice QED, Nucl. Phys. B 323 (1989) 631.

[38] R. Narayanan and J. Nishimura, Parity-invariant lattice regularization of a threedimensional gauge-fermion system, Nucl. Phys. B 508 (1997) 371 hep-th/9703109.

[39] S. Okubo and Y. Tosa, Further study of global gauge anomalies of simple groups, Phys. Rev. D 40 (1989) 1925.

[40] R. Holman and T.W. Kephart, Global anomalies in Yang-Mills theories in higher dimensions, Phys. Lett. B 167 (1986) 417.

[41] S. Okubo, H. Zhang, Y. Tosa and R.E. Marshak, SU(N) global gauge anomalies in even dimensions, Phys. Rev. D 37 (1988) 1655.

[42] O. Bär and I. Campos, Global anomalies in chiral gauge theories on the lattice, Nucl. Phys. B $\mathbf{5 8 1}(2000) 499$ hep-lat/0001025.

[43] L. Alvarez-Gaumé and E. Witten, Gravitational anomalies, Nucl. Phys. B 234 (1984) 269.

[44] T.W. Chiu, The spectrum and topological charge of exactly massless fermions on the lattice, Phys. Rev. D 58 (1998) 074511 hep-lat/9804016;

K. Fujikawa, Relation $\operatorname{Tr} \gamma_{5}=0$ and the index theorem in lattice gauge theory, Phys. Rev. D 60 (1999) 074505 hep-lat/9904007. 
